\title{
Rudin's Dowker space in the extension with a Suslin tree
}

\author{
by
}

Teruyuki Yorioka (Shizuoka)

\begin{abstract}
We introduce a generalization of a Dowker space constructed from a Suslin tree by Mary Ellen Rudin, and the rectangle refining property for forcing notions, which modifies the one for partitions due to Paul B. Larson and Stevo Todorčević and is stronger than the countable chain condition. It is proved that Martin's Axiom for forcing notions with the rectangle refining property implies that every generalized Rudin space constructed from Aronszajn trees is non-Dowker, and that the same can be forced with a Suslin tree. Moreover, we consider generalized Rudin spaces constructed with some types of non-Aronszajn $\omega_{1}$-trees under the Proper Forcing Axiom.
\end{abstract}

1. Introduction. In [7], Dowker investigated characterizations of countable paracompactness of Hausdorff normal spaces, and he asked if every Hausdorff normal space is countably paracompact.

The first counterexample is due to Mary E. Rudin [15]. She proved that if the Suslin Hypothesis fails (that is, there exists a Suslin tree), then there exists a Hausdorff normal space which is not countably paracompact, and moreover it is of size $\aleph_{1}$. A Hausdorff normal space which is not countably paracompact is called a Dowker space. In [16], Rudin exhibited a ZFCexample of a Dowker space which however is quite big, and she asked whether there exists a Dowker space of size $\aleph_{1}$ from only ZFC. This is still unknown. (See e.g. [19].) The best known ZFC-example of a Dowker space is of size $\min \left\{2^{\aleph_{0}}, \aleph_{\omega+1}\right\}$ and is obtained by combining the results of Balogh [1] and Kojman-Shelah [9].

In this paper, we generalize Rudin's Dowker space constructed from a Suslin tree. We will show that Martin's Axiom for forcing notions with the rectangle refining property implies that every generalized Rudin space

2000 Mathematics Subject Classification: Primary 03E35; Secondary 54A35, 54D15, 54D20, 54G20.

Key words and phrases: Dowker spaces, Martin's Axiom and its fragments, the rectangle refining property.

Supported by Grant-in-Aid for JSPS Fellow, No. 18840022, Ministry of Education, Culture, Sports, Science and Technology. 
constructed with Aronszajn trees is normal and countably paracompact (hence is not Dowker), and show that it is consistent that there exists a Suslin tree which forces the same result. Moreover, we consider generalized Rudin spaces with non-Aronszajn $\omega_{1}$-trees under the Proper Forcing Axiom.

1.1. A generalization of Rudin's Dowker space. Basically, we follow the standard notation of set theory, as given in [8]. An ordinal $\alpha$ is the set of all ordinals smaller than $\alpha$, and for ordinals $\alpha$ and $\beta,[\alpha, \beta)$ is the interval of ordinals between $\alpha$ and $\beta$ including $\alpha$, that is, $[\alpha, \beta)=\{\gamma \in \beta ; \alpha \leq \gamma\}$. Lim stands for the class of limit ordinals.

To simplify notation, in this paper except for a part of $\S 3$, an $\omega_{1}$-tree $T$ is a subset of $\omega^{<\omega_{1}}$ such that $s \leq_{T} t$ iff $s \subseteq t$ for all $s, t \in T$, and for every $t \in T, \operatorname{lv}(t)$, called the height (or level) of $t$, is just the length of $t$ (that is, $T$ is closed under initial segments). For nodes $s$ and $t$ in $T$, let

$$
\begin{aligned}
\Delta_{T}(s, t) & =\Delta(s, t) \\
& := \begin{cases}\min \{\alpha \in \min \{\operatorname{lv}(s), \operatorname{lv}(t)\} ; s(\alpha) \neq t(\alpha)\} \\
\operatorname{if} s \text { and } t \text { are incomparable in } T, \\
\min \{\operatorname{lv}(s), \operatorname{lv}(t)\} & \text { otherwise. }\end{cases}
\end{aligned}
$$

We note that for $s$ and $t$ in $T, s \uparrow \Delta(s, t)=t \uparrow \Delta(s, t)$, where $s \uparrow \Delta(s, t)$ is the function $s$ restricted to the domain $\Delta(s, t)$, and let $s \wedge t=s \uparrow \Delta(s, t)$. For an $\omega_{1}$-tree $T, \alpha \in \omega_{1}$ and $t \in T$, let $T_{\alpha}$ be the set of the $\alpha$ th level nodes in $T$, and define

$$
T_{\leq \alpha}:=\bigcup_{\gamma \in \alpha+1} T_{\gamma} \text { and } T_{\geq \alpha}:=\bigcup_{\gamma \in\left[\alpha, \omega_{1}\right)} T_{\gamma}
$$

Let $T\left\lceil t\right.$ be the set of nodes $s$ such that $s \geq_{T} t$. In this paper, we assume that every tree $T$ is Hausdorff, that is, if $\alpha$ is a limit ordinal and $s$ and $t$ are different nodes of $T_{\alpha}$, then

$$
\left\{u \in T ; u<_{T} s\right\} \neq\left\{u \in T ; u<_{T} t\right\} .
$$

Let $\left\langle T^{n} ; n \in \omega\right\rangle$ be a decreasing sequence of $\omega_{1}$-trees, that is, each $T^{n}$ is an $\omega_{1}$-tree and $T^{n} \supseteq T^{n+1}$ for every $n \in \omega$. For this sequence, we consider a sequence $\left\langle\pi^{n} ; n \in \omega \backslash\{0\}\right\rangle$ of functions such that for each $n \in \omega \backslash\{0\}$,

(p1) $\pi^{n}$ is a function with the domain $\bigcup_{\alpha \in \omega_{1} \cap \operatorname{Lim}}\left(T^{n}\right)_{\alpha}$ such that for every $t \in T^{n}$ with a limit level, $\pi^{n}(t)$ is an infinite subset of $\left(T^{n-1}\right)_{\mathrm{Iv}(t)}$,

(p2) for any $t \in T^{n}$ with a limit level, the set $\left\{\Delta(s, t) ; s \in \pi^{n}(t)\right\}$ is cofinal in $\operatorname{lv}(t)$ and $t \notin \pi^{n}(t)$,

(p3) for any distinct nodes $s$ and $t$ in $\operatorname{dom}\left(\pi^{n}\right), \pi^{n}(s) \cap \pi^{n}(t)=\emptyset$. 
In this paper, $\omega_{1}$-trees are always considered to be sufficiently branching $\left({ }^{1}\right)$ to be able to take such functions $\pi^{n}$. Then we define a topological space $\mathfrak{X}\left(\left\langle T^{n}, \pi^{n} ; n \in \omega\right\rangle\right)$ on the set $\bigcup_{n \in \omega} T^{n} \times\{n\}$ as follows.

For $i \in \omega, s \in T^{i}$ and $\beta \in \omega_{1}$, if $s$ is of a limit height and $i=0$, then we let

$$
N(\langle s, 0\rangle, \beta):=\left\{u \in T^{0} ; u \leq_{T^{0}} s \& \beta<\operatorname{lv}(u)\right\} \times\{0\} ;
$$

if $s$ is of a limit height and $i>0$, then

$$
\begin{aligned}
N(\langle s, i\rangle, \beta):= & \left(\left\{u \in T^{i} ; u \leq_{T^{i}} s \& \beta<\operatorname{lv}(u)\right\} \times\{i\}\right) \\
& \cup\left(\left\{u \in \pi^{i}(s) ; \Delta(u, s)>\beta\right\} \times\{i-1\}\right) ;
\end{aligned}
$$

and otherwise, that is, if $s$ is of a successor height, then $N(\langle s, i\rangle, \beta):=$ $\{\langle s, i\rangle\}$. Note that each $N(\langle s, i\rangle, \beta)$ is countable. A subset $U$ of $\bigcup_{n \in \omega} T^{n} \times\{n\}$ is open iff for any $x=\langle s, i\rangle \in U$, there is $\beta \in \operatorname{Iv}(s)$ such that $N(x, \beta)$ $\subseteq U$. The following are basic observations $\left({ }^{2}\right)$ about the topological space $\mathfrak{X}\left(\left\langle T^{n}, \pi^{n} ; n \in \omega\right\rangle\right)$.

OBSERVATION 1.1. Suppose that $\left\langle T^{n} ; n \in \omega\right\rangle$ is a decreasing sequence of $\omega_{1}$-trees and $\left\langle\pi^{n} ; n \in \omega \backslash\{0\}\right\rangle$ is a sequence of functions with the properties (p1-3) $\left(\right.$ for $\left.\left\langle T^{n} ; n \in \omega\right\rangle\right)$. Then the topological space $\mathfrak{X}\left(\left\langle T^{n}, \pi^{n} ; n \in \omega\right\rangle\right)$ has the following properties:

1. For each $k \in \omega$, the set $\bigcup_{n \in k} T^{n} \times\{n\}$ is open.

2. For each $\delta \in \omega_{1}$, the set $\bigcup_{n \in \omega}\left(T^{n}\right)_{\geq \delta+1} \times\{n\}$ is clopen.

3. For all $x \in \bigcup_{n \in \omega} T^{n} \times\{n\}$ and $\beta \in \omega_{1}, N(x, \beta)$ is closed.

4. For each $t \in T^{0}$ with successor height, the set

$$
\bigcup_{n \in \omega}\left(\left(T^{0}\lceil t) \cap T^{n}\right) \times\{n\}\right.
$$

is clopen.

5. Every singleton is closed.

6. If $C$ and $D$ are closed subsets and $C$ is countable, then they are separated by two disjoint open sets.

Therefore the topological space $\mathfrak{X}\left(\left\langle T^{n}, \pi^{n} ; n \in \omega\right\rangle\right)$ is Hausdorff and regular. The following is essentially due to Rudin.

Theorem 1.2 (Rudin [15]). Suppose that $\left\langle T^{n} ; n \in \omega\right\rangle$ is a decreasing sequence of $\omega_{1}$-trees and $\left\langle\pi^{n} ; n \in \omega \backslash\{0\}\right\rangle$ is a sequence of functions with (p1-3). If all $T^{n}$ are Suslin trees (that is, $T^{0}$ is Suslin), then $\mathfrak{X}\left(\left\langle T^{n}, \pi^{n}\right.\right.$; $n \in \omega\rangle)$ is a Dowker space.

$\left({ }^{1}\right)$ If each $T^{n}$ is non-atomic, then we can find $\pi^{n}$ 's which are defined at some club level nodes. This is enough to define Rudin's original Dowker space. However, to simplify notation, we assume conditions (p1-3) in this paper.

$\left({ }^{2}\right)$ All of them are stated in [15]. In particular, the last statement was proved in the last half of $\S 6$ there. 
In fact, Rudin's original Dowker space is of the form $\mathfrak{X}\left(\left\langle T^{n}, \pi^{n} ; n \in \omega\right\rangle\right)$ where all $T^{n}$ are the same Suslin tree, all $\pi^{n}$ are the same function, and for each $n \in \omega \backslash\{0\}$,

(p4) for any $t \in T^{n}$ with a limit level, if $s$ and $s^{\prime}$ are different nodes in $\pi^{n}(t)$, then $\Delta(s, t) \neq \Delta\left(s^{\prime}, t\right)$, and the set $\left\{\Delta(s, t) ; s \in \pi^{n}(t)\right\}$ is of order-type $\omega$ with respect to $\in$ and convergent to $\operatorname{lv}(t)$ (hence $\left.t \notin \pi^{n}(t)\right)$.

The following theorem is due to Dowker and is frequently used to verify that a given space is Dowker.

TheOrEm 1.3 (Dowker [7]). Suppose that $\mathfrak{X}$ is a Hausdorff normal space. The following are equivalent:

(D0) $\mathfrak{X}$ is not countably paracompact.

(D1) There exists a sequence $\left\langle C_{n} ; n \in \omega\right\rangle$ of closed subsets of $\mathfrak{X}$ such that

- $C_{n+1} \subseteq C_{n}$ for every $n \in \omega$,

- $\bigcap_{n \in \omega} C_{n}=\emptyset$,

- $\bigcap_{n \in \omega} U_{n} \neq \emptyset$ for every sequence $\left\langle U_{n} ; n \in \omega\right\rangle$ of open subsets of $\mathfrak{X}$ such that $C_{n} \subseteq U_{n}$ for all $n \in \omega$.

Therefore if a Hausdorff space does not satisfy (D1), it is not a Dowker space whether it is normal or not.

1.2. The rectangle refining property. A partition $K_{0} \cup K_{1}$ on $\left[\omega_{1}\right]^{2}$ has the rectangle refining property if for any uncountable subsets $I$ and $J$ of $\omega_{1}$, there are uncountable subsets $I^{\prime}$ and $J^{\prime}$ of $I$ and $J$ respectively such that if $\alpha \in I^{\prime}, \beta \in J^{\prime}$ and $\alpha<\beta$, then $\{\alpha, \beta\} \in K_{0}$. This notion was introduced by Larson-Todorčević in [14]. We note that the rectangle refining property is stronger than the countable chain condition (ccc for short) for partitions. (See e.g. [22].) We introduce the rectangle refining property for forcing notions as follows. A forcing notion $\mathbb{P}$ has the rectangle refining property if $\mathbb{P}$ is uncountable and there exists a property $P$, which is defined from $\mathbb{P}$ (and other parameters used to define $\mathbb{P}$ ), and is absolute between any transitive models with the same $\omega_{1}$ and such that any uncountable subset of $\mathbb{P}$ has an uncountable subset with the property $P$, and for any uncountable subsets $I$ and $J$ of $\mathbb{P}$, if $I \cup J$ has the property $P$, then there are uncountable subsets $I^{\prime}$ and $J^{\prime}$ of $I$ and $J$ respectively such that every member of $I^{\prime}$ is compatible in $\mathbb{P}$ with every member of $J^{\prime}$. In this paper, we call such $P$ a suitable refinement for $\mathbb{P}$. We also note that this is stronger than the countable chain condition for forcing notions.

A typical example of a forcing notion with the rectangle refining property is as follows. Let $K_{0} \cup K_{1}$ be a partition on $\left[\omega_{1}\right]^{2}$. Let $\mathbb{P}$ be a forcing 
notion which consists of finite $K_{0}$-homogeneous subsets of $\omega_{1}$, ordered by reverse inclusion. This generically adds an uncountable $K_{0}$-homogeneous set if $K_{0} \cup K_{1}$ is a ccc partition. We notice that if $K_{0} \cup K_{1}$ has the rectangle refining property, then so does $\mathbb{P}$, and a suitable refinement is just a $\Delta$-system refinement. For other examples, see [23]. Let $\mathcal{K}_{2}$ (rec) be the statement that for every two-colored partition on $\left[\omega_{1}\right]^{2}$ with the rectangle refining property, there exists an uncountable $K_{0}$-homogeneous set (defined in [14]), and let $\mathrm{MA}_{\aleph_{1}}$ (rec) be Martin's Axiom for $\aleph_{1}$-many dense sets of forcing notions with the rectangle refining property. It follows from the above example that $\mathrm{MA}_{\aleph_{1}}$ (rec) implies $\mathcal{K}_{2}(\mathrm{rec})$. By the same argument in [23], we can show that it is consistent that $M A_{\aleph_{1}}$ (rec) holds but $M A_{\aleph_{1}}$ fails, so $M A_{\aleph_{1}}$ (rec) is strictly weaker than $\mathrm{MA}_{\aleph_{1}}$.

In Section 2, we introduce two types of forcing notions to prove the following two theorems.

THEOREM 1. $\mathrm{MA}_{\aleph_{1}}$ (rec) implies that if $\left\langle T^{n} ; n \in \omega\right\rangle$ is a decreasing sequence of Aronszajn trees and $\left\langle\pi^{n} ; n \in \omega \backslash\{0\}\right\rangle$ is a sequence of functions with (p1-3), then the space $\mathfrak{X}\left(\left\langle T^{n}, \pi^{n} ; n \in \omega\right\rangle\right)$ does not satisfy (D1).

THEOREM 2. $\mathrm{MA}_{\aleph_{1}}(\mathrm{rec})$ implies that if $\left\langle T^{n} ; n \in \omega\right\rangle$ is a decreasing sequence of Aronszajn trees and $\left\langle\pi^{n} ; n \in \omega \backslash\{0\}\right\rangle$ is a sequence of functions with (p1-4), then the space $\mathfrak{X}\left(\left\langle T^{n}, \pi^{n} ; n \in \omega\right\rangle\right)$ is normal.

Therefore we conclude that $\mathrm{MA}_{\aleph_{1}}$ (rec) implies that if $\left\langle T^{n} ; n \in \omega\right\rangle$ is a decreasing sequence of Aronszajn trees and $\left\langle\pi^{n} ; n \in \omega \backslash\{0\}\right\rangle$ is a sequence of functions with (p1-4), then the topology $\mathfrak{X}\left(\left\langle T^{n}, \pi^{n} ; n \in \omega\right\rangle\right)$ is normal and countably paracompact.

In Section 3, we prove the following theorem, which gives a new example that may be forced by a Suslin tree. (This relates to a question in $[12, \S 6]$.)

THEOREM 3. It is consistent that there exists a Suslin tree which forces that

1. if $\left\langle T^{n} ; n \in \omega\right\rangle$ is a decreasing sequence of Aronszajn trees and $\left\langle\pi^{n}\right.$; $n \in \omega \backslash\{0\}\rangle$ is a sequence of functions with (p1-3), then the space $\mathfrak{X}\left(\left\langle T^{n}, \pi^{n} ; n \in \omega\right\rangle\right)$ does not satisfy (D1),

2. if $\left\langle T^{n} ; n \in \omega\right\rangle$ is a decreasing sequence of Aronszajn trees and $\left\langle\pi^{n}\right.$; $n \in \omega \backslash\{0\}\rangle$ is a sequence of functions with (p1-4), then the space $\mathfrak{X}\left(\left\langle T^{n}, \pi^{n} ; n \in \omega\right\rangle\right)$ is normal.

Notice that we do not prove in this paper that a Suslin tree may force $M A_{\aleph_{1}}(\mathrm{rec})$. It is a deep question whether $\mathrm{MA}_{\aleph_{1}}(\mathrm{rec})$ is strictly stronger than $\mathcal{K}_{2}$ (rec), similarly to other problems on fragments of Martin's Axiom as e.g. in $[22, \S 7]$ (see also $[12,13,23])$.

In Section 4, we consider generalized Rudin spaces constructed with nonAronszajn trees. We obtain the following two results: 
THEOREM 4. $\mathrm{MA}_{\aleph_{1}}$ (rec) implies that if $\left\langle T^{n} ; n \in \omega\right\rangle$ is a decreasing sequence of $\omega_{1}$-trees and $\left\langle\pi^{n} ; n \in \omega \backslash\{0\}\right\rangle$ is a sequence of functions with (p1-3) such that $T^{0}$ has at most countably many cofinal chains, then the space $\mathfrak{X}\left(\left\langle T^{n}, \pi^{n} ; n \in \omega\right\rangle\right)$ does not satisfy (D1).

Theorem 5. The Proper Forcing Axiom implies that if $\left\langle T^{n} ; n \in \omega\right\rangle$ is a decreasing sequence of $\omega_{1}$-trees with the properties (u1-2) (see Section 4.2 for the definition) and $\left\langle\pi^{n} ; n \in \omega \backslash\{0\}\right\rangle$ is a sequence of functions with (p1-3), then the space $\mathfrak{X}\left(\left\langle T^{n}, \pi^{n} ; n \in \omega\right\rangle\right)$ does not satisfy (D1).

To end this section, we give a key combinatorial property of Aronszajn trees, which is used in many places, mainly in Section 2. It follows from the next lemma that if $T$ is an Aronszajn tree, then the forcing notion adding an uncountable antichain through $T$ by finite approximations has the rectangle refining property.

Lemma $1.4([23])$. Suppose that $T$ is an Aronszajn tree, and $I$ and $J$ are uncountable subsets of $T$. Then there exist incomparable nodes $u$ and $v$ in $T$ such that the sets $\left\{s \in I ; u<_{T} s\right\}$ and $\left\{s \in J ; v<_{T} s\right\}$ are both uncountable.

\section{Generalized Rudin spaces constructed with Aronszajn trees}

2.1. Proof of Theorem 1. Throughout this subsection, we suppose that $\left\langle T^{n} ; n \in \omega\right\rangle$ is a decreasing sequence of Aronszajn trees and $\left\langle\pi^{n} ; n \in \omega \backslash\{0\}\right\rangle$ is a sequence of functions with (p1-3).

Let $C$ be a closed subset of $\mathfrak{X}\left(\left\langle T^{n}, \pi^{n} ; n \in \omega\right\rangle\right)$. We define a forcing notion

$$
\mathrm{D}\left(\left\langle T^{n}, \pi^{n} ; n \in \omega\right\rangle, C\right),
$$

or $\mathrm{D}(C)$ for short, which consists of pairs $\langle A, h\rangle$ such that

1. $A$ is a finite subset of $\bigcup_{n \in \omega} T^{n} \times\{n\} \backslash C$,

2. $h$ is a finite partial function from $\bigcup_{n \in \omega} T^{n} \times\{n\}$ into $\omega_{1}$,

3. for every $x=\langle s, i\rangle \in \operatorname{dom}(h), h(x) \in \operatorname{Iv}(s)$ and $N(x, h(x)) \cap A=\emptyset$; moreover, if $s$ is a successor level, then $h(x)=\operatorname{lv}(s)-1$,

ordered by extensions, that is, for conditions $\langle A, h\rangle$ and $\left\langle A^{\prime}, h^{\prime}\right\rangle$,

$$
\langle A, h\rangle \leq_{\mathrm{D}(C)}\left\langle A^{\prime}, h^{\prime}\right\rangle: \Leftrightarrow A \supseteq A^{\prime} \& h \supseteq h^{\prime} .
$$

We notice that for any $x \in \bigcup_{n \in \omega} T^{n} \times\{n\}$, the set

$$
\{\langle A, h\rangle \in \mathrm{D}(C) ; x \in A \cup \operatorname{dom}(h)\}
$$

is dense in $\mathrm{D}(C)$. (The proof is essentially the same as the proof of the regularity of $\mathfrak{X}\left(\left\langle T^{n}, \pi^{n} ; n \in \omega\right\rangle\right)$.) So $\mathrm{D}(C)$ generically adds an open set $U$ covering $C$ and a subset $W$ of $\bigcup_{n \in \omega} T^{n} \times\{n\}$ such that $U$ and $W$ are disjoint and $U \cup W$ covers the space.

Lemma 2.1. $\mathrm{D}\left(\left\langle T^{n}, \pi^{n} ; n \in \omega\right\rangle, C\right)$ has the rectangle refining property. 
Proof. First, we exhibit a suitable refinement for this forcing notion. Let $\left\{\left\langle A_{\xi}, h_{\xi}\right\rangle ; \xi \in \omega_{1}\right\}$ be an uncountable family in $\mathrm{D}(C)$. By shrinking the family if necessary, we may assume that

- both $\left\{A_{\zeta} ; \zeta \in \omega_{1}\right\}$ and $\left\{\operatorname{dom}\left(h_{\zeta}\right) ; \zeta \in \omega_{1}\right\}$ form $\Delta$-systems with roots $R$ and $S$ respectively,

- for every $\zeta$ and $\mu$ in $\omega_{1}, h_{\zeta}\left\lceil S=h_{\mu}\lceil S\right.$,

- the size of all $A_{\zeta} \backslash R$ is the same, say $k$, and if $A_{\zeta} \backslash R=\left\{x_{\zeta, i} ; i \in k\right\}$ then each $x_{\zeta, i}$ equals $\left\langle s_{\zeta, i}, m_{i}\right\rangle$ for some $m_{i}$,

- the size of all $\operatorname{dom}\left(h_{\zeta}\right) \backslash S$ is the same, say $l$, and if $\operatorname{dom}\left(h_{\zeta}\right) \backslash S=$ $\left\{y_{\zeta, j} ; j \in l\right\}$ then each $y_{\zeta, j}$ is $\left\{t_{\zeta, j}, n_{j}\right\}$ for some $n_{j}$,

- there exists $\delta$ such that both $R$ and $S$ are subsets of $\bigcup_{n \in \omega}\left(T^{n}\right)_{\leq \delta+1} \times$ $\{n\}$ and for $j \in l$ and $\zeta, \mu \in \omega_{1}$,

$$
\left\{\langle s \uparrow(\delta+1), m\rangle ;\langle s, m\rangle \in A_{\zeta}\right\}=\left\{\langle s \uparrow(\delta+1), m\rangle ;\langle s, m\rangle \in A_{\mu}\right\}
$$

and $t_{\zeta, j} \uparrow(\delta+1)=t_{\mu, j}\lceil(\delta+1)$,

- there exists a subset $L$ of $l$ such that for any $j \in L$, all the $h_{\zeta}\left(y_{\zeta, j}\right)$ are the same $\alpha_{j}$, and for all $\zeta, \mu \in \omega_{1}, t_{\zeta, j}\left\lceil\left(\alpha_{j}+1\right)=t_{\eta, j}\left\lceil\left(\alpha_{j}+1\right)\right.\right.$, and for any $j \in l \backslash L$, the set $\left\{h_{\zeta}\left(y_{\zeta, j}\right) ; \zeta \in \omega_{1}\right\}$ is uncountable.

This is a suitable refinement for our forcing notion.

Suppose that $I_{0}$ and $I_{1}$ are disjoint uncountable subsets of $\omega_{1}$, and a pair of families $\left\{\left\langle A_{\xi}, h_{\xi}\right\rangle ; \xi \in I_{0}\right\}$ and $\left\{\left\langle A_{\eta}, h_{\eta}\right\rangle ; \eta \in I_{1}\right\}$ of uncountable subsets of $\mathrm{D}(C)$ forms a suitable refinement, that is, $\left\{\left\langle A_{\zeta}, h_{\zeta}\right\rangle ; \zeta \in I_{0} \cup I_{1}\right\}$ is as above.

Since each $T^{n}$ is Aronszajn, by using Lemma 1.4 finitely many times, there are uncountable subsets $I_{0}^{\prime}$ and $I_{1}^{\prime}$ of $I_{0}$ and $I_{1}$ respectively and a set $\left\{w_{e, i}, z_{e, j} ; e \in\{0,1\} \& i \in k \& j \in l\right\}$ of nodes of $T^{0}$ such that

- for any $\zeta<\mu$ in $I_{0}^{\prime} \cup I_{1}^{\prime}$,

$$
\begin{aligned}
\max & \left\{\operatorname{lv}(s) ;\langle s, m\rangle \in A_{\zeta} \cup \operatorname{dom}\left(h_{\zeta}\right)\right\} \\
& <\min \left(\left\{\operatorname{lv}\left(s_{\mu, i}\right) ; i \in k\right\} \cup\left\{h_{\mu}\left(y_{\mu, j}\right) ; j \in l \backslash L\right\} \cup\left\{\operatorname{lv}\left(t_{\mu, j}\right) ; j \in L\right\}\right),
\end{aligned}
$$

- for every $e \in\{0,1\}, \zeta \in I_{e}^{\prime}, i \in k$ and $j \in l$, we have $w_{e, i} \leq_{T^{0}} s_{\zeta, i}$ and $z_{e, j} \leq_{T^{0}} t_{\zeta, j}$, and $w_{e, i}$ and $z_{1-e, j}$ are incomparable in $T^{0}$.

Then for all $\xi \in I_{0}^{\prime}$ and $\eta \in I_{1}^{\prime}$,

$$
\left(A_{\xi} \cup A_{\eta}\right) \cap\left(\bigcup_{x \in \operatorname{dom}\left(h_{\xi}\right)} N\left(x, h_{\xi}(x)\right) \cup \bigcup_{x \in \operatorname{dom}\left(h_{\eta}\right)} N\left(x, h_{\eta}(x)\right)\right)=\emptyset,
$$

hence $\left\langle A_{\xi}, h_{\xi}\right\rangle$ and $\left\langle A_{\eta}, h_{\eta}\right\rangle$ are compatible in $\mathrm{D}(C)$.

Suppose that $\left\langle C_{\nu} ; \nu \in \omega\right\rangle$ is a decreasing sequence of closed sets with empty intersection. Then the finite support product $\prod_{\nu \in \omega} \mathrm{D}\left(C_{\nu}\right)$ also has the rectangle refining property, and for any $x \in \bigcup_{n \in \omega} T^{n} \times\{n\}$ and $v \in \omega$, 
the sets

$$
\left\{\left\langle\left\langle A_{\nu}, h_{\nu}\right\rangle ; \nu \in \sigma\right\rangle \in \prod_{\nu \in \omega} \mathrm{D}\left(C_{\nu}\right) ; v \in \sigma \& x \in A_{v} \cup \operatorname{dom}\left(h_{v}\right)\right\}
$$

and

$$
\left\{\left\langle\left\langle A_{\nu}, h_{\nu}\right\rangle ; \nu \in \sigma\right\rangle \in \prod_{\nu \in \omega} \mathrm{D}\left(C_{\nu}\right) ; \exists \mu \in \sigma\left(x \in A_{\mu}\right)\right\}
$$

are both dense in $\prod_{\nu \in \omega} \mathrm{D}\left(C_{\nu}\right)$. Hence for a $\prod_{\nu \in \omega} \mathrm{D}\left(C_{\nu}\right)$-generic filter $G$, letting

$$
U_{v}:=\bigcup\left\{N\left(x, h_{\nu}(x)\right) ; \exists\left\langle\left\langle A_{\nu}, h_{\nu}\right\rangle ; \nu \in \sigma\right\rangle \in G\left(v \in \sigma \& x \in \operatorname{dom}\left(h_{v}\right)\right)\right\}
$$

and

$$
W_{v}:=\bigcup\left\{A_{v} ; \exists\left\langle\left\langle A_{\nu}, h_{\nu}\right\rangle ; \nu \in \sigma\right\rangle \in G(v \in \sigma)\right\}
$$

for each $v \in \omega$, we have:

- $U_{v}$ is an open set which covers $C_{v}$,

- $W_{v}$ is disjoint from $U_{v}$ and $U_{v} \cup W_{v}$ covers the whole space,

- $\bigcup_{\nu \in \omega} W_{\nu}$ covers the whole space, hence $\bigcap_{\nu \in \omega} U_{\nu}$ is empty.

That is, $\prod_{\nu \in \omega} \mathrm{D}\left(C_{\nu}\right)$ adds a counterexample to (D1) for the sequence $\left\langle C_{\nu} ; \nu \in \omega\right\rangle$. Therefore Theorem 1 follows from the above lemma.

2.2. Proof of Theorem 2. Throughout this subsection, we suppose that $\left\langle T^{n} ; n \in \omega\right\rangle$ is a decreasing sequence of Aronszajn trees and $\left\langle\pi^{n} ; n \in \omega \backslash\{0\}\right\rangle$ is a sequence of functions with (p1-4).

Suppose that $C$ and $D$ are disjoint closed subsets of $\bigcup_{n \in \omega} T^{n} \times\{n\}$. Then we define a forcing notion

$$
\mathrm{N}\left(\left\langle T^{n}, \pi^{n} ; n \in \omega\right\rangle, C, D\right),
$$

or $\mathrm{N}(C, D)$ for short, which consists of pairs $\langle c, d\rangle$ of finite partial functions from $\bigcup_{n \in \omega} T^{n} \times\{n\}$ into $\omega_{1}$ such that:

1. for any $\langle s, i\rangle \in \operatorname{dom}(c)$, if $s$ has a limit level, then $c(\langle s, i\rangle) \in \operatorname{lv}(s)$, otherwise $c(\langle s, i\rangle)=\operatorname{lv}(s)-1$,

2. for any $\langle t, j\rangle \in \operatorname{dom}(d)$, if $t$ has a limit level, then $d(\langle t, j\rangle) \in \operatorname{lv}(t)$, otherwise $d(\langle t, j\rangle)=\operatorname{lv}(t)-1$,

3. $\left(C \cup \bigcup_{x \in \operatorname{dom}(c)} N(x, c(x))\right) \cap\left(D \cup \bigcup_{x \in \operatorname{dom}(d)} N(x, d(x))\right)=\emptyset$,

ordered by extensions, that is, for conditions $\langle c, d\rangle$ and $\left\langle c^{\prime}, d^{\prime}\right\rangle$,

$$
\langle c, d\rangle \leq_{\mathrm{N}(C, D)}\left\langle c^{\prime}, d^{\prime}\right\rangle: \Leftrightarrow c \supseteq c^{\prime} \& d \supseteq d^{\prime} .
$$

Note that for each $x \in \bigcup_{n \in \omega} T^{n} \times\{n\}$, the set

$$
\{\langle c, d\rangle \in \mathrm{N}(C, D) ; x \in \operatorname{dom}(c) \cup \operatorname{dom}(d)\}
$$


is dense in $\mathrm{N}(C, D)$. The proof is also essentially the same as the proof of the regularity of $\mathfrak{X}\left(\left\langle T^{n}, \pi^{n} ; n \in \omega\right\rangle\right)$. From the genericity argument for these dense sets, for any $\mathrm{N}(C, D)$-generic filter $G$,

$$
\bigcup\{N(x, c(x)) ; \exists\langle c, d\rangle \in G(x \in \operatorname{dom}(c))\}
$$

and

$$
\bigcup\{N(x, d(x)) ; \exists\langle c, d\rangle \in G(x \in \operatorname{dom}(d))\}
$$

are disjoint open sets which separate $C$ and $D$. So Theorem 2 follows from the next lemma.

Lemma 2.2. $\mathrm{N}\left(\left\langle T^{n}, \pi^{n} ; n \in \omega\right\rangle, C, D\right)$ has the rectangle refining property.

Proof. First, we exhibit a suitable refinement for this forcing notion. Suppose that $\left\{\left\langle c_{\zeta}, d_{\zeta}\right\rangle ; \zeta \in \omega_{1}\right\}$ is an uncountable family in $\mathrm{N}(C, D)$. By shrinking the family if necessary, we may assume that there are subsets $R$ and $S$ of $\bigcup_{n \in \omega} T^{n} \times\{n\}$ such that $\left\{\operatorname{dom}\left(c_{\zeta}\right) ; \zeta \in \omega_{1}\right\}$ and $\left\{\operatorname{dom}\left(d_{\zeta}\right) ; \zeta \in \omega_{1}\right\}$ are both $\Delta$-systems with roots $R$ and $S$ respectively. Moreover, we may assume that $c_{\zeta}\left\lceil R=c_{\mu}\left\lceil R\right.\right.$ and $d_{\zeta}\left\lceil S=d_{\mu}\left\lceil S\right.\right.$ for all $\zeta, \mu \in \omega_{1}$. By shrinking the family more if necessary, we may also assume that

- the size of all $\operatorname{dom}\left(c_{\zeta}\right) \backslash R$ is the same, say $k$, the size of all $\operatorname{dom}\left(d_{\zeta}\right) \backslash S$ is the same, say $l$, and if $\operatorname{dom}\left(c_{\zeta}\right) \backslash R=\left\{x_{\zeta, i} ; i \in k\right\}$ and $\operatorname{dom}\left(d_{\zeta}\right) \backslash S=$ $\left\{y_{\zeta, j} ; j \in l\right\}$, then each $x_{\zeta, i}$ is $\left\langle s_{\zeta, i}, m_{i}\right\rangle$ and each $y_{\zeta, j}$ is $\left\langle t_{\zeta, j}, n_{j}\right\rangle$ for some $m_{i}$ and $n_{j}$,

- there exists $\delta$ such that both $R$ and $S$ are subsets of $\bigcup_{n \in \omega}\left(T^{n}\right)_{\leq \delta+1} \times$ $\{n\}$, and $s_{\zeta, i} \uparrow(\delta+1)=s_{\mu, i} \uparrow(\delta+1)$ and $t_{\zeta, j} \uparrow(\delta+1)=t_{\mu, j} \uparrow(\delta+1)$ for all $i \in k, j \in l$ and $\zeta, \mu \in \omega_{1}$,

- there exist $K \subseteq k$ and $L \subseteq l$ such that

- for any $i \in K$, the $c_{\zeta}\left(x_{\zeta, i}\right)$ are the same $\alpha_{i}$, and $s_{\zeta, i} \uparrow\left(\alpha_{i}+1\right)=$ $s_{\mu, i} \uparrow\left(\alpha_{i}+1\right)$ for all $\zeta, \mu \in \omega_{1}$,

- for any $i \in k \backslash K$, the set $\left\{c_{\zeta}\left(x_{\zeta, i}\right) ; \zeta \in \omega_{1}\right\}$ is uncountable,

- for any $j \in L$, the $d_{\zeta}\left(y_{\zeta, j}\right)$ are the same $\beta_{j}$, and $t_{\zeta, j} \uparrow\left(\beta_{j}+1\right)=$ $t_{\mu, j} \uparrow\left(\beta_{j}+1\right)$ for all $\zeta, \mu \in \omega_{1}$,

- for any $j \in l \backslash L$, the set $\left\{d_{\zeta}\left(y_{\zeta, i}\right) ; \zeta \in \omega_{1}\right\}$ is uncountable.

This is a suitable refinement for this forcing notion.

Suppose that $I_{0}$ and $I_{1}$ are disjoint uncountable subsets of $\omega_{1}$ and a pair of families $\left\{\left\langle c_{\xi}, d_{\xi}\right\rangle ; \xi \in I_{0}\right\}$ and $\left\{\left\langle c_{\eta}, d_{\eta}\right\rangle ; \eta \in I_{1}\right\}$ of uncountable subsets of $\mathrm{N}(C, D)$ forms a suitable refinement, that is, $\left\{\left\langle c_{\zeta}, d_{\zeta}\right\rangle ; \zeta \in I_{0} \cup I_{1}\right\}$ is as above.

Since each $T^{n}$ is Aronszajn and $\left\langle\pi^{n} ; n \in \omega \backslash\{0\}\right\rangle$ satisfies (p4), by using Lemma 1.4 finitely many times we can find uncountable subsets $I_{0}^{\prime}$ and $I_{1}^{\prime}$ 
of $I_{0}$ and $I_{1}$ respectively and sets $\left\{w_{e, i}, z_{e, j} ; e \in\{0,1\} \& i \in k \& j \in l\right\}$ of nodes of $T^{0}$ such that

- for any $\zeta<\mu$ in $I_{0}^{\prime} \cup I_{1}^{\prime}$,

$$
\begin{aligned}
& \max \{\operatorname{lv}(s) ;\langle s, m\rangle\left.\in \operatorname{dom}\left(c_{\zeta}\right) \cup \operatorname{dom}\left(d_{\zeta}\right)\right\} \\
&<\min \left\{c_{\mu}\left(x_{\mu, i}\right), d_{\mu}\left(y_{\mu, j}\right) ; i \in k \backslash K \& j \in l \backslash L\right\},
\end{aligned}
$$

and for every $e \in\{0,1\}$,

- for all $i \in k \backslash K, j \in l \backslash L$ and $\zeta \in I_{e}^{\prime}$, we have $w_{e, i}<_{T^{m_{i}}} s_{\zeta, i} \uparrow c_{\zeta}\left(x_{\zeta, i}\right)$ and $z_{e, j}<_{T^{n_{j}}} t_{\zeta, j}\left\lceil d_{\zeta}\left(y_{\zeta, j}\right)\right.$,

- for all $i \in K, j \in L$ and $\zeta \in I_{e}^{\prime}$, we have $\operatorname{lv}\left(w_{e, i}\right) \geq \beta_{j}+1, w_{e, i}<_{T^{m_{i}}} s_{\zeta, i}$, $\operatorname{lv}\left(z_{e, j}\right) \geq \alpha_{i}+1$ and $z_{e, j}<_{T^{n_{j}}} t_{\zeta, j}$,

- for all $i \in k$ and $j \in l, w_{e, i}$ and $z_{1-e, j}$ are incomparable in $T^{0}$,

- for all $i \in k \backslash K$ and $j \in L$, if $m_{i}=n_{j}-1$, then either $\Delta\left(w_{e, i}, z_{1-e, j}\right)$ is not in the set

$$
\left\{\Delta\left(t_{\mu, j}, y\right) ; \mu \in I_{1-e}^{\prime} \& y \in \pi^{n_{j}}\left(t_{\mu, j}\right)\right\}
$$

or there exist $r_{1-e, i, j} \in T^{m_{i}}$ and $a_{\mu, 1-e, i, j} \in \pi^{n_{j}}\left(t_{\mu, j}\right)$ for each $\mu \in$ $I_{1-e}^{\prime}$ such that for all $\mu \in I_{1-e}^{\prime}$ we have $\Delta\left(w_{e, i}, z_{1-e, j}\right)=$ $\Delta\left(t_{\mu, j}, a_{\mu, 1-e, i, j}\right), r_{1-e, i, j}<_{T^{m_{i}}} a_{\mu, 1-e, i, j}$, and $r_{1-e, i, j}$ and $w_{e, i}$ are incomparable in $T^{m_{i}}$,

- for all $j \in l \backslash L$ and $i \in K$, if $n_{j}=m_{i}-1$, then either $\Delta\left(z_{e, j}, w_{1-e, i}\right)$ is not in the set

$$
\left\{\Delta\left(s_{\mu, i}, z\right) ; \mu \in I_{1-e}^{\prime} \& z \in \pi^{m_{i}}\left(s_{\mu, i}\right)\right\},
$$

or there exist $u_{1-e, j, i} \in T^{n_{j}}$ and $b_{\mu, 1-e, j, i} \in \pi^{m_{i}}\left(s_{\mu, i}\right)$ for each $\mu \in$ $I_{1-e}^{\prime}$ such that for all $\mu \in I_{1-e}^{\prime}$ we have $\Delta\left(z_{e, j}, w_{1-e, i}\right)=$ $\Delta\left(s_{\mu, i}, b_{\mu, 1-e, j, i}\right), u_{1-e, j, i}<_{T^{n_{j}}} b_{\mu, 1-e, j, i}$, and $u_{1-e, j, i}$ and $z_{e, j}$ are incomparable in $T^{n_{j}}$.

The last two conditions guarantee that for all $i \in k \backslash K$ and $j \in L$, if $m_{i}=n_{j}-1$, then $w_{e, i}$ is incomparable in $T^{m_{i}}$ with any member of $\bigcup_{\mu \in I_{1-e}^{\prime}} \pi^{n_{j}}\left(t_{\mu, j}\right)$, and for all $j \in l \backslash L$ and $i \in K$, if $n_{j}=m_{i}-1$, then $z_{e, j}$ is incomparable in $T^{n_{j}}$ with any member of $\bigcup_{\mu \in I_{1-e}^{\prime}} \pi^{m_{i}}\left(s_{\mu, i}\right)$. So by our $\Delta$-system refinement, if $e \in\{0,1\}, \xi \in I_{e}^{\prime}, \eta \in I_{1-e}^{\prime}, i \in k$ and $j \in l$, then

$$
N\left(x_{\xi, i}, c_{\xi}\left(x_{\xi, i}\right)\right) \cap N\left(y_{\eta, j}, d_{\eta}\left(y_{\eta, j}\right)\right)=\emptyset .
$$

Hence for all $\xi \in I_{0}^{\prime}$ and $\eta \in I_{1}^{\prime}$, the pair $\left\langle c_{\xi} \cup c_{\eta}, d_{\xi} \cup d_{\eta}\right\rangle$ is a condition of $\mathrm{N}(C, D)$, so $\left\langle c_{\xi}, d_{\xi}\right\rangle$ and $\left\langle c_{\eta}, d_{\eta}\right\rangle$ are compatible in $\mathrm{N}(C, D)$. 


\section{A Suslin tree may force that every generalized Rudin space} constructed with Aronszajn trees is non-Dowker. Throughout this section, we suppose that $T$ is a coherent Suslin tree which consists of functions in $\omega^{<\omega_{1}}$ and is closed under finite modifications. That is, for any $s$ and $t$ in $T$, the set

$$
\{\alpha \in \min \{\operatorname{lv}(s), \operatorname{lv}(t)\} ; s(\alpha) \neq t(\alpha)\}
$$

is finite, and for any $s \in T$ and $t \in \omega^{\operatorname{lv}(s)}$, if $\{\alpha \in \operatorname{lv}(s) ; s(\alpha) \neq t(\alpha)\}$ is finite, then also $t \in T$. We note that $\diamond$, or adding a Cohen real, builds a coherent Suslin tree. A coherent Suslin tree has canonical commutative isomorphisms. Let $s$ and $t$ be nodes in $T$ with the same height. Then we define a function $\psi_{s, t}$ from $T\left\lceil s\right.$ into $T\left\lceil t\right.$ such that for each $v \in T$ with $v \geq_{T} s$,

$$
\psi_{s, t}(v):=t \Upsilon(v\lceil[\operatorname{lv}(s), \operatorname{lv}(v))),
$$

the concatenation of $t$ and $v\left\lceil[\operatorname{lv}(s), \operatorname{lv}(v))\right.$. Note that $\psi_{s, t}$ is an isomorphism, and if $s, t, u$ are nodes in $T$ with the same level, then $\psi_{s, t}, \psi_{t, u}$ and $\psi_{s, u}$ commute. (On coherent Suslin trees, see e.g. [10, 13].)

3.1. Proof of Theorem 3(1). Throughout this subsection, we suppose that $\left\langle\dot{T}^{n} ; n \in \omega\right\rangle$ is a sequence of $T$-names for Aronszajn trees and $\left\langle\dot{\pi}_{n}\right.$; $n \in \omega \backslash\{0\}\rangle$ is a sequence of $T$-names for functions with (p1-3). Moreover, we suppose that it is forced that for each $n \in \omega$ and each $\alpha \in \omega_{1}$, the set $\left(\dot{T}^{n}\right)_{\alpha}$ is a subset of $\left[\lambda_{\alpha}, \lambda_{\alpha}+\omega\right)$, where $\lambda_{\alpha}$ is the $\alpha$ th limit ordinal.

For a countable ordinal $\alpha$, we let $\operatorname{lv}(\alpha):=\sup (\operatorname{Lim} \cap(\alpha+1))$, that is, $\operatorname{lv}(\alpha)$ is the unique limit ordinal $\lambda$ such that $\alpha \in[\lambda, \lambda+\omega)$. We notice that in general $\operatorname{lv}(\alpha)$ is not equal to the level of the tree in the usual sense, but they are equal at club many levels.

Let $\dot{C}_{\nu}$, for $\nu \in \omega$, be $T$-names for closed subsets of $\mathfrak{X}\left(\left\langle\dot{T}^{n}, \dot{\pi}^{n} ; n \in \omega\right\rangle\right)$ such that it is forced that $\left\langle\dot{C}_{\nu} ; \nu \in \omega\right\rangle$ is a decreasing sequence of closed sets with empty intersection. Since $T$ is ccc, we can find a club $Z$ on $\omega_{1}$ such that for every $\gamma \in Z, \lambda_{\gamma}=\gamma$, and for any $\alpha, \beta \in \gamma$ and $m, n, \nu \in \omega$, every node in $T_{\gamma}$ decides the statements " $\alpha \in \dot{T}$ " ", " $\alpha \leq_{\dot{T}^{n}} \beta$ ", " $\langle\alpha, \beta\rangle \in \dot{\pi}^{n "}$, and " $\langle\alpha, m\rangle \in \dot{C}_{\nu}$ ". One of the important observations to show Lemma 3.4 (and Lemma 3.7) below is that, since $T$ is ccc, for any $\alpha \in \omega_{1}$ and $n \in \omega$, the set

$$
\begin{aligned}
& \left\{\left\{\beta \in \omega_{1} ; u \Vdash_{T} " \beta<_{\dot{T}^{n}} \alpha "\right\} ;\right. \\
& \left.u \in T \& u \text { decides all levels of the tree } \dot{T}^{n} \text { below } \alpha\right\}
\end{aligned}
$$

is countable.

For a finite partial function $c$ from $\omega_{1} \times \omega$ into $\omega_{1}, s \in T$ and $\delta \in \omega_{1}$, if for every $\langle\alpha, m\rangle \in \operatorname{dom}(c), s \Vdash_{T} " \alpha \in \dot{T}^{m}$ " and $c(\langle\alpha, m\rangle) \in \operatorname{lv}(\alpha)$, and 
$\sup (Z \cap(\delta+1)) \leq \operatorname{lv}(s)$, then we let

$$
\begin{array}{r}
M(c, s, \delta):=\bigcup_{\langle\alpha, m\rangle \in \operatorname{dom}(c)}\left\{\beta \in \sup (Z \cap(\delta+1)) ; s \Vdash_{T} " \beta \leq_{\dot{T}^{m}} \alpha "\right. \\
\quad \& \operatorname{lv}(\beta)>c(\langle\alpha, m\rangle)\} \times\{m\} .
\end{array}
$$

Note that for such $c, s$ and $\delta$,

$$
s \Vdash_{T} \text { " } M(c, s, \delta) \text { is a closed subset of } \mathfrak{X}\left(\left\langle\dot{T}^{n}, \dot{\pi}^{n} ; n \in \omega\right\rangle\right) \text { ". }
$$

We define a forcing notion

$$
g_{T} \mathrm{D}\left(\left\langle\dot{T}^{n}, \dot{\pi}^{n} ; n \in \omega\right\rangle,\left\langle\dot{C}_{\nu} ; \nu \in \omega\right\rangle, Z\right),
$$

or $g \mathrm{D}\left(\left\langle\dot{C}_{\nu} ; \nu \in \omega\right\rangle, Z\right)$ for short, which consists of the triples $\langle\sigma, f, g\rangle$ such that:

1. $\sigma$ is a finite subset of $\omega$,

2. $f$ and $g$ are functions, $\operatorname{dom}(f)=\operatorname{dom}(g)$ and $\operatorname{dom}(f)$ is a finite subset of $T$ closed under $\wedge$,

3. for each $t \in \operatorname{dom}(f), f(t)$ is a finite sequence of finite subsets of the set $\sup (Z \cap(\operatorname{lv}(t)+1)) \times \omega$ with a support $\sigma$, say $f(t)=\langle f(t, \nu) ; \nu \in \sigma\rangle$,

4. for each $t \in \operatorname{dom}(f), g(t)$ is a finite sequence of finite partial functions from $\sup (Z \cap(\operatorname{lv}(t)+1)) \times \omega$ into $\omega_{1}$ with support $\sigma$, say $g(t)=$ $\langle g(t, \nu) ; \nu \in \sigma\rangle$,

5. $t \Vdash_{T}$ " $\langle\langle f(t, \nu), g(t, \nu)\rangle ; \nu \in \sigma\rangle \in \prod_{\nu \in \omega} \mathrm{D}\left(\left\langle\dot{T}^{n}, \dot{\pi}^{n} ; n \in \omega\right\rangle, \dot{C}_{\nu}\right)$ ",

6. for all $t$ and $t^{\prime}$ in $\operatorname{dom}(f)$, if $t^{\prime}<_{T} t$, then for each $\nu \in \sigma$,

$$
t \Vdash_{T} "\langle f(t, \nu), g(t, \nu)\rangle \leq_{\mathrm{D}\left(\dot{C}_{\nu}\right)}\left\langle f\left(t^{\prime}, \nu\right), g\left(t^{\prime}, \nu\right)\right\rangle "
$$

and if

$$
\begin{aligned}
& \left\{\operatorname{lv}(\alpha) ;\langle\alpha, m\rangle \in \bigcup_{\nu \in \sigma_{t}} f(t, \nu) \backslash f\left(t^{\prime}, \nu\right)\right. \text { or } \\
& \left.\quad\langle\alpha, m\rangle \in \bigcup_{\nu \in \sigma_{t}} \operatorname{dom}(g(t, \nu)) \backslash \operatorname{dom}\left(g\left(t^{\prime}, \nu\right)\right), \text { for some } m \in \omega\right\} \neq \emptyset
\end{aligned}
$$

then

$$
\begin{aligned}
& \max \left\{\operatorname{lv}(\alpha) ;\langle\alpha, m\rangle \in \bigcup_{\nu \in \sigma_{t^{\prime}}} f\left(t^{\prime}, \nu\right)\right. \text { or } \\
& \left.\qquad \alpha, m\rangle \in \bigcup_{\nu \in \sigma_{t^{\prime}}} \operatorname{dom}\left(g\left(t^{\prime}, \nu\right)\right) \text {, for some } m \in \omega\right\} \\
& <\min \left\{\operatorname{lv}(\alpha) ;\langle\alpha, m\rangle \in \bigcup_{\nu \in \sigma_{t}} f(t, \nu) \backslash f\left(t^{\prime}, \nu\right)\right. \text { or } \\
& \left.\langle\alpha, m\rangle \in \bigcup_{\nu \in \sigma_{t}} \operatorname{dom}(g(t, \nu)) \backslash \operatorname{dom}\left(g\left(t^{\prime}, \nu\right)\right) \text {, for some } m \in \omega\right\},
\end{aligned}
$$


7. for all $s, t \in \operatorname{dom}(f)$ and $\nu \in \sigma$,

$$
f(s \wedge t, \nu)=f(s, \nu) \cap f(t, \nu) \cap(\sup (Z \cap(\Delta(s, t)+1)) \times \omega)
$$

and

$$
\begin{aligned}
\operatorname{dom}(g & (s \wedge t, \nu)) \\
\quad & =\operatorname{dom}(g(s, \nu)) \cap \operatorname{dom}(g(t, \nu)) \cap(\sup (Z \cap(\Delta(s, t)+1)) \times \omega),
\end{aligned}
$$

8. for all $s, t \in \operatorname{dom}(f)$ and $\nu \in \sigma$,

$$
\begin{aligned}
((f(s, \nu) \cup f(t, \nu)) & \cap \Delta(s, t)) \\
& \cap(M(g(s, \nu), s, \Delta(s, t)) \cup M(g(t, \nu), t, \Delta(s, t)))=\emptyset .
\end{aligned}
$$

For conditions $\langle\sigma, f, g\rangle$ and $\left\langle\sigma^{\prime}, f^{\prime}, g^{\prime}\right\rangle$ in $g \mathrm{D}\left(\left\langle\dot{C}_{\nu} ; \nu \in \omega\right\rangle, Z\right)$, we define

$$
\begin{aligned}
&\langle\sigma, f, g\rangle \leq_{g \mathrm{D}\left(\left\langle\dot{C}_{\nu} ; \nu \in \omega\right\rangle, Z\right)}\left\langle\sigma^{\prime}, f^{\prime}, g^{\prime}\right\rangle \\
&: \Leftrightarrow \sigma \supseteq \sigma^{\prime} \& \operatorname{dom}(f) \supseteq \operatorname{dom}\left(f^{\prime}\right) \& \forall \nu \in \sigma^{\prime} \forall t \in \operatorname{dom}\left(f^{\prime}\right) \\
&\left(t \Vdash_{T} "\langle f(t, \nu), g(t, \nu)\rangle \leq_{\mathrm{D}\left(\dot{C}_{\nu}\right)}\left\langle f^{\prime}(t, \nu), g^{\prime}(t, \nu)\right\rangle "\right) .
\end{aligned}
$$

Proposition 3.1. For each $t \in T$, the set

$$
\left\{\langle\sigma, f, g\rangle \in g_{T} \mathrm{D}\left(\left\langle\dot{T}^{n}, \dot{\pi}^{n} ; n \in \omega\right\rangle,\left\langle\dot{C}_{\nu} ; \nu \in \omega\right\rangle, Z\right) ; t \in \operatorname{dom}(f)\right\}
$$

is dense.

Proof. Let $\langle\sigma, f, g\rangle \in g \mathrm{D}\left(\left\langle\dot{C}_{n} ; n \in \omega\right\rangle, Z\right)$.

If there is $s \in \operatorname{dom}(f)$ such that $t \leq_{T} s$, then for each $\nu \in \sigma$, let

$$
\begin{aligned}
& \left.f^{\prime}(t, \nu):=f(s, \nu) \cap(\sup (Z \cap(\operatorname{lv}(t)+1)) \times \omega)\right), \\
& g^{\prime}(t, \nu):=g(s, \nu)\lceil(\operatorname{dom}(g(s, \nu)) \cap \sup (Z \cap(\operatorname{lv}(t)+1)) \times \omega)) .
\end{aligned}
$$

Then

$$
\left\langle\sigma, f \cup\left\{\left\langle t,\left\langle f^{\prime}(t, \nu) ; \nu \in \sigma\right\rangle\right\rangle\right\}, g \cup\left\{\left\langle t,\left\langle g^{\prime}(t, \nu) ; \nu \in \sigma\right\rangle\right\rangle\right\}\right\rangle
$$

is a condition of $g \mathrm{D}\left(\left\langle\dot{C}_{\nu} ; \nu \in \omega\right\rangle, Z\right)$ and an extension of $\langle\sigma, f, g\rangle$.

Suppose that $t \mathbb{Z}_{T} s$ for all $s \in \operatorname{dom}(f)$. Then there exists $s \in \operatorname{dom}(f)$ such that $\Delta(t, u) \leq \Delta(t, s)$ for any $u \in \operatorname{dom}(f)$. For each $\nu \in \sigma$ we let

$$
\begin{aligned}
& \left.f^{\prime}(s \wedge t, \nu):=f(s, \nu) \cap(\sup (Z \cap(\Delta(t, s)+1)) \times \omega)\right), \\
& g^{\prime}(s \wedge t, \nu):=g(s, \nu)\lceil(\operatorname{dom}(g(s, \nu)) \cap \sup (Z \cap(\Delta(t, s)+1)) \times \omega)) .
\end{aligned}
$$

Then

$$
\left\langle\sigma, f \cup\left\{\left\langle s \wedge t,\left\langle f^{\prime}(s \wedge t, \nu) ; \nu \in \sigma\right\rangle\right\rangle\right\}, g \cup\left\{\left\langle s \wedge t,\left\langle g^{\prime}(s \wedge t, \nu) ; \nu \in \sigma\right\rangle\right\rangle\right\}\right\rangle
$$
is a condition of $g \mathrm{D}\left(\left\langle\dot{C}_{\nu} ; \nu \in \omega\right\rangle, Z\right)$ and an extension of $\langle\sigma, f, g\rangle$.

Proposition 3.2. For $t \in T,\langle\alpha, m\rangle \in \omega_{1} \times \omega$ and $v \in \omega$, the set

$$
\left\{\langle\sigma, f, g\rangle \in g_{T} \mathrm{D}\left(\left\langle\dot{T}^{n}, \dot{\pi}^{n} ; n \in \omega\right\rangle,\left\langle\dot{C}_{\nu} ; \nu \in \omega\right\rangle, Z\right) ;\right.
$$

$v \in \sigma \& \exists s \in \operatorname{dom}(f)\left(t \leq_{T} s\right.$

$\&\left(\right.$ either $s \Vdash_{T}$ " $\alpha \notin \dot{T}^{m}$ " or $\left.\left.\left.\langle\alpha, m\rangle \in f(s, v) \cup \operatorname{dom}(g(s, v))\right)\right)\right\}$

is dense in $g_{T} \mathrm{D}\left(\left\langle\dot{T}^{n}, \dot{\pi}^{n} ; n \in \omega\right\rangle,\left\langle\dot{C}_{\nu} ; \nu \in \omega\right\rangle, Z\right)$. 
Proof. Let $\langle\sigma, f, g\rangle \in g \mathrm{D}\left(\left\langle\dot{C}_{\nu} ; \nu \in \omega\right\rangle, Z\right)$. From the previous proposition, we may assume that $t \in \operatorname{dom}(f)$ and there exists $s \in T$ such that $t \leq_{T} s, \operatorname{lv}(u) \leq \operatorname{lv}(s)$ for every $u \in \operatorname{dom}(f)$, and $\operatorname{lv}(\alpha)<\sup (Z \cap(\operatorname{lv}(s)+1))$. Moreover, we may assume that $v \in \sigma$. By the property of $Z, s$ decides the statement " $\alpha \in \dot{T}^{m}$ ". If $s \Vdash_{T}$ " $\alpha \notin \dot{T}^{m}$ ", then $\langle\sigma, f, g\rangle$ is in the above set, so now we suppose that $s \Vdash " \alpha \in \dot{T}^{m}$ ".

We enumerate the set

$$
\{u \in \operatorname{dom}(f) ; \operatorname{lv}(\alpha) \in \sup (Z \cap(\Delta(u, s)+1))\}
$$

as $\left\langle u_{i} ; i \in k\right\rangle$. Note that for any $j \in k$ and $u \in \operatorname{dom}(f), \quad$ if $\operatorname{lv}(\alpha) \in \sup (Z \cap$ $\left.\left(\Delta\left(u_{j}, u\right)+1\right)\right)$, then $u$ is also in the set $\left\{u_{i} ; i \in k\right\}$, and $u \Vdash_{T}$ " $\alpha \in \dot{T}^{m}$ ". If for some $i \in k,\langle\alpha, m\rangle \in f\left(u_{i}, v\right) \cup \operatorname{dom}\left(g\left(u_{i}, v\right)\right)$, then $\langle\sigma, f, g\rangle$ is in the above set, so now we suppose that $\langle\alpha, m\rangle \notin f\left(u_{i}, v\right) \cup \operatorname{dom}\left(g\left(u_{i}, v\right)\right)$ for any $i \in k$. Then we recursively pick $\gamma_{i} \in \operatorname{lv}(\alpha)$ for $i \in k$ such that for each $i \in k$, $\gamma_{i} \leq \gamma_{i+1}$ and

$$
u_{i} \Vdash_{T} "\left\langle f\left(u_{i}, v\right), g\left(u_{i}, v\right) \cup\left\{\left\langle\langle\alpha, m\rangle, \gamma_{i}\right\rangle\right\}\right\rangle \in \mathrm{D}\left(\dot{C}_{v}\right) ",
$$

and for any $u \in \operatorname{dom}(f)$,

$$
f(u, v) \cap M\left(g\left(u_{i}\right) \cup\left\{\left\langle\langle\alpha, m\rangle, \gamma_{i}\right\rangle\right\}, u_{i}, \Delta\left(u_{i}, u\right)\right)=\emptyset .
$$

Then the triple

$$
\begin{aligned}
\left\langle\sigma, f,\left(g \left\lceil\left(\operatorname { d o m } ( g ) \backslash \left(\left\{u_{i} ; i \in k\right\}\right.\right.\right.\right.\right. & \times\{v\}))) \\
\cup & \left.\left\{\left\langle u_{i}, g\left(u_{i}\right) \cup\left\{\left\langle\langle\alpha, m\rangle, \gamma_{k-1}\right\rangle\right\}\right\rangle ; i \in k\right\}\right\rangle
\end{aligned}
$$

is a condition of $g \mathrm{D}\left(\left\langle\dot{C}_{\nu} ; \nu \in \omega\right\rangle, Z\right)$ and an extension of $\langle\sigma, f, g\rangle$.

Proposition 3.3. For $t \in T,\langle\alpha, m\rangle \in \omega_{1} \times \omega$, the set

$$
\begin{aligned}
\{\langle\sigma, f, g\rangle \in & g_{T} \mathrm{D}\left(\left\langle\dot{T}^{n}, \dot{\pi}^{n} ; n \in \omega\right\rangle,\left\langle\dot{C}_{\nu} ; \nu \in \omega\right\rangle, Z\right) ; \\
& \exists s \in \operatorname{dom}(f)\left(t \leq_{T} s\right. \\
& \left.\left.\&\left(\text { either } s \Vdash_{T} \text { " } \alpha \notin \dot{T}^{m} \text { " or } \exists \nu \in \sigma(\langle\alpha, m\rangle \in f(s, \nu))\right)\right)\right\}
\end{aligned}
$$

is dense in $g_{T} \mathrm{D}\left(\left\langle\dot{T}^{n}, \dot{\pi}^{n} ; n \in \omega\right\rangle,\left\langle\dot{C}_{\nu} ; \nu \in \omega\right\rangle, Z\right)$.

Proof. Let $\langle\sigma, f, g\rangle \in g \mathrm{D}\left(\left\langle\dot{C}_{\nu} ; \nu \in \omega\right\rangle, Z\right)$. We may assume that $t \in$ $\operatorname{dom}(f)$ and there exists $s \in T$ such that $t \leq_{T} s, \operatorname{lv}(u) \leq \operatorname{lv}(s)$ for every $u \in \operatorname{dom}(f)$, and $\operatorname{lv}(\alpha)<\sup (Z \cap(\operatorname{lv}(s)+1))$. By the property of $Z, s$ decides the statement " $\alpha \in \dot{T}^{m}$ ". If $s \Vdash_{T}$ " $\alpha \notin \dot{T}^{m}$ ", then $\langle\sigma, f, g\rangle$ is in the above set, so now we suppose that $s \Vdash$ " $\alpha \in \dot{T}^{m}$ ". Then we can find $\nu \in \omega \backslash \sigma$ such that $s \Vdash_{T}$ " $\langle\alpha, m\rangle \notin \dot{C}_{\nu}$ ". So we can find the desired extension of $\langle\sigma, f, g\rangle$.

Assume that $g \mathrm{D}\left(\left\langle\dot{C}_{\nu} ; \nu \in \omega\right\rangle, Z\right) \times T$ is ccc. (Then the forcing notion $g \mathrm{D}\left(\left\langle\dot{C}_{\nu} ; \nu \in \omega\right\rangle, Z\right)$ is also ccc.) We denote the ground model by $\mathbf{V}$ and let $G$ be a $g \mathrm{D}\left(\left\langle\dot{C}_{\nu} ; \nu \in \omega\right\rangle, Z\right)$-generic filter over $\mathbf{V}$, and $H$ a $T$-generic filter 
over $\mathbf{V}[G]$. Then we let

$$
\begin{aligned}
G_{H}:=\left\{p \in \prod_{\nu \in \omega} \mathrm{D}\left(\dot{C}_{\nu}[H]\right) ; \exists\langle\sigma, f, g\rangle \in G \exists t \in \operatorname{dom}(f) \cap H\right. \\
\left.\quad\left(t \Vdash_{T} "\langle f(t, \nu), g(t, \nu) ; \nu \in \sigma\rangle \leq_{\prod_{\nu \in \omega} \mathrm{D}\left(\dot{C}_{\nu}[H]\right)} p "\right)\right\} .
\end{aligned}
$$

$\left(\dot{C}_{\nu}[H]\right.$ is the interpretation of $\dot{C}_{\nu}$ in $\mathbf{V}[G][H]$ by $H$.) By the definition of $g \mathrm{D}\left(\left\langle\dot{C}_{\nu} ; \nu \in \omega\right\rangle, Z\right), G_{H}$ is a filter. By the previous propositions, we note that in $\mathbf{V}[G]$,

- for each $\nu \in \omega$ and $\langle\alpha, m\rangle \in \omega_{1} \times \omega$, the set

$\left\{s \in T\right.$; either $s \Vdash_{T}$ " $\alpha \notin \dot{T}^{m}$ "

$$
\text { or } \exists\langle\sigma, f, g\rangle \in G(\nu \in \sigma \&\langle\alpha, m\rangle \in f(s, \nu) \cup \operatorname{dom}(g(s, \nu)))\}
$$

is dense in $T$,

- for each $\langle\alpha, m\rangle \in \omega_{1} \times \omega$, the set

$$
\begin{aligned}
\left\{s \in T ; \text { either } s \Vdash_{T}\right. & \text { " } \alpha \notin \dot{T}^{m} " \\
& \text { or } \exists\langle\sigma, f, g\rangle \in G \exists \nu \in \sigma(\langle\alpha, m\rangle \in f(s, \nu))\}
\end{aligned}
$$

is dense in $T$.

In $\mathbf{V}[G][H]$, for each $v \in \omega$, we let

$$
\begin{aligned}
& U_{v}:=\bigcup\left\{N\left(x, h_{v}(x)\right) ;\right.\left.\exists\left\langle\left\langle A_{\nu}, h_{\nu}\right\rangle ; \nu \in \sigma\right\rangle \in G_{H}\left(v \in \sigma \& x \in \operatorname{dom}\left(h_{v}\right)\right)\right\}, \\
& W_{v}:=\bigcup\left\{A_{v} ; \exists\left\langle\left\langle A_{\nu}, h_{\nu}\right\rangle ; \nu \in \sigma\right\rangle \in G_{H}(v \in \sigma)\right\} .
\end{aligned}
$$

Then for each $v \in \omega$,

- $U_{v}$ is an open set which covers $C_{v}$,

- $W_{v}$ is disjoint from $U_{v}$ and $U_{v} \cup W_{v}$ covers the whole space,

- $\bigcup_{\nu \in \omega} W_{\nu}$ covers the whole space, hence $\bigcap_{\nu \in \omega} U_{\nu}$ is empty.

Lemma 3.4. $g_{T} \mathrm{D}\left(\left\langle\dot{T}^{n}, \dot{\pi}^{n} ; n \in \omega\right\rangle,\left\langle\dot{C}_{\nu} ; \nu \in \omega\right\rangle, Z\right) \times T$ satisfies the countable chain condition.

Proof. Let $\left\{\left\langle\left\langle\sigma_{\xi}, f_{\xi}, g_{\xi}\right\rangle, t_{\xi}\right\rangle ; \xi \in \omega_{1}\right\}$ be an uncountable subset of $g \mathrm{D}\left(\left\langle\dot{C}_{\nu} ;\right.\right.$ $\nu \in \omega\rangle, Z) \times T$. By strengthening each condition if necessary, we may assume that there are $\sigma \in[\omega]^{<\aleph_{0}}$ and $\delta_{\xi} \in \omega_{1}$ for each $\xi \in \omega_{1}$ such that for each $\xi \in \omega_{1}$,

- $\sigma_{\xi}=\sigma$,

- $t_{\xi} \in \operatorname{dom}\left(f_{\xi}\right) \cap T_{\delta_{\xi}}$ and every node in $\operatorname{dom}\left(f_{\xi}\right)$ is of height $\leq \delta_{\xi}$,

- for any $t \in \operatorname{dom}\left(f_{\xi}\right)$, there exists $t^{\prime} \in \operatorname{dom}\left(f_{\xi}\right) \cap T_{\delta_{\xi}}$ such that $t \leq_{T} t^{\prime}$.

By shrinking the subset and strengthening each condition again if necessary, we may also assume that there exists $\gamma_{0} \in \omega_{1}$ such that 
- $\gamma_{0}<\delta_{0}$ and if $\xi<\eta$ in $\omega_{1}$, then $\delta_{\xi}<\delta_{\eta}$,

- $\left\{\operatorname{dom}\left(f_{\xi}\right) ; \xi \in \omega_{1}\right\}$ is a $\Delta$-system with a root $R$ such that $R \subseteq T_{\leq \gamma_{0}}$ and if $\xi<\eta$ in $\omega_{1}$, then $f_{\xi}\left\lceil R=f_{\eta}\left\lceil R\right.\right.$ and $g_{\xi}\left\lceil R=g_{\eta}\left\lceil R\right.\right.$, and $\delta_{\xi}<\operatorname{lv}(s)$ for any $s \in \operatorname{dom}\left(f_{\eta}\right) \backslash R$,

- for each $\nu \in \sigma$,

- $\left\{\bigcup_{t \in \operatorname{dom}\left(f_{\xi}\right)} f_{\xi}(t, \nu) ; \xi \in \omega_{1}\right\}$ is a $\Delta$-system with a root $S_{0}^{\nu} \subseteq \gamma_{0} \times \omega$, and $\delta_{\xi}<\operatorname{lv}(\varepsilon)$ for any $\xi<\eta$ in $\omega_{1}$ and $\langle\varepsilon, i\rangle \in \bigcup_{t \in \operatorname{dom}\left(f_{\eta}\right)} f_{\eta}(t, \nu) \backslash S_{0}^{\nu}$,

- $\left\{\bigcup_{t \in \operatorname{dom}\left(g_{\xi}\right)} \operatorname{dom}\left(g_{\xi}(t, \nu)\right) ; \xi \in \omega_{1}\right\}$ is a $\Delta$-system with a root $S_{1}^{\nu} \subseteq$ $\gamma_{0} \times \omega$, and if $\xi<\eta$ in $\omega_{1}$, then $\delta_{\xi}<\operatorname{lv}(\varepsilon)$ for any $\langle\varepsilon, i\rangle \in$ $\bigcup_{t \in \operatorname{dom}\left(g_{\eta}\right)} \operatorname{dom}\left(g_{\eta}(t, \nu)\right) \backslash S_{1}^{\nu}$,

- there is a strictly increasing sequence $\left\langle\gamma_{\xi} ; \xi \in \omega_{1} \backslash\{0\}\right\rangle$ of countable ordinals such that for every $\xi \in \omega_{1}$, the set

$$
\left\{\zeta \in \omega_{1} ; \exists s, t \in \operatorname{dom}\left(f_{\xi}\right) \cap T_{\delta_{\xi}}\left(\zeta \in \delta_{\xi} \& s(\zeta) \neq t(\zeta)\right)\right\}
$$

is a subset of $\gamma_{0} \cup\left[\gamma_{\xi}, \gamma_{\xi+1}\right)$,

- $\left|\operatorname{dom}\left(f_{\xi}\right) \cap T_{\delta_{\xi}}\right|=: n$ for all $\xi \in \omega_{1}$, say

$$
\operatorname{dom}\left(f_{\xi}\right) \cap T_{\delta_{\xi}}=\left\{s_{0}^{\xi}, s_{1}^{\xi}, \ldots, s_{n-1}^{\xi}\right\},
$$

and $t_{\xi}=s_{0}^{\xi}$, and there exists $m \leq n$ such that for any $\xi \in \omega_{1}$ and $i \in n$, there is a unique $j \in m$ such that $s_{i}^{\xi}\left\lceil\gamma_{\xi}=s_{j}^{\xi}\left\lceil\gamma_{\xi}\right.\right.$ and $s_{j}^{\xi}\left\lceil\left[\gamma_{\xi}, \gamma_{\xi+1}\right)=\right.$ $t_{\xi} \uparrow\left[\gamma_{\xi}, \gamma_{\xi+1}\right)$, and for all $i \in n$ and $\xi, \eta \in \omega_{1}, s_{i}^{\xi}\left\lceil\gamma_{0}=s_{i}^{\eta}\left\lceil\gamma_{0}\right.\right.$,

- for any $\xi, \eta \in \omega_{1}, \nu \in \sigma$ and $i \in n, g_{\xi}\left(s_{i}^{\xi}, \nu\right)\left\lceil S_{1}^{\nu}=g_{\eta}\left(s_{i}^{\eta}, \nu\right)\left\lceil S_{1}^{\nu}\right.\right.$,

- for all $\nu \in \sigma$ and $i \in n$,

- the size of all $f_{\xi}\left(s_{i}^{\xi}, \nu\right) \backslash S_{0}^{\nu}$ is the same, say $k_{i}^{\nu}$, the size of all $\operatorname{dom}\left(g_{\xi}\left(s_{i}^{\xi}, \nu\right)\right) \backslash S_{1}^{\nu}$ is the same, say $l_{i}^{\nu}$, and we have $f_{\xi}\left(s_{i}^{\xi}, \nu\right) \backslash S_{0}^{\nu}=$ $\left\{\left\langle\alpha_{\nu, \imath}^{\xi}, a_{\nu, \imath}\right\rangle ; \imath \in k_{i}^{\nu}\right\}$ and $\operatorname{dom}\left(g_{\xi}\left(s_{i}^{\xi}, \nu\right)\right) \backslash S_{1}^{\nu}=\left\{\left\langle\beta_{\nu, \jmath}^{\xi}, b_{\nu, \jmath}\right\rangle ; \jmath \in l_{i}^{\nu}\right\}$ for some $a_{\nu, \imath}$ and $b_{\nu, \jmath}$,

- if $\xi<\eta$ in $\omega_{1}$ and $\imath \in k_{i}^{\nu}$, then

$$
\begin{aligned}
\left\{\varepsilon \in \gamma_{0}+1 ; s_{i}^{\xi} \Vdash_{T} " \varepsilon<_{\dot{T}^{a_{\nu, \jmath}}} \alpha_{\nu, \jmath}^{\xi} "\right\} & \\
& =\left\{\varepsilon \in \gamma_{0}+1 ; s_{i}^{\eta} \Vdash_{T} " \varepsilon<_{\dot{T}^{a_{\nu, \jmath}}} \alpha_{\nu, \jmath}^{\eta} "\right\},
\end{aligned}
$$

- there is $L_{i}^{\nu} \subseteq l_{i}^{\nu}$ such that

$*$ if $\xi<\eta$ in $\omega_{1}$ and $\jmath \in l_{i}^{\nu} \backslash L_{i}^{\nu}$, then $\delta_{\xi}<g_{\eta}\left(s_{i}^{\eta}, \nu\right)\left(\left\langle\beta_{\nu, \jmath}^{\eta}, b_{\nu, \jmath}\right\rangle\right)$ and

$$
\begin{aligned}
\left\{\varepsilon \in \gamma_{0}+1 ; s_{i}^{\xi} \Vdash_{T} " \varepsilon\right. & \left.<\dot{T}^{b_{\nu, j}} \beta_{\nu, \jmath}^{\xi} "\right\} \\
& =\left\{\varepsilon \in \gamma_{0}+1 ; s_{i}^{\eta} \Vdash_{T} " \varepsilon<_{\dot{T}^{b_{\nu, \jmath}}} \beta_{\nu, j}^{\eta} "\right\},
\end{aligned}
$$

$*$ if $\xi, \eta \in \omega_{1}$ and $\jmath \in L_{i}^{\nu}$, then

$$
g_{\xi}\left(s_{i}^{\xi}, \nu\right)\left(\left\langle\beta_{\nu, \jmath}^{\xi}, b_{\nu, \jmath}\right\rangle\right)=g_{\eta}\left(s_{i}^{\eta}, \nu\right)\left(\left\langle\beta_{\nu, \jmath}^{\eta}, b_{\nu, \jmath}\right\rangle\right)
$$


and

$$
\begin{aligned}
& \left\{\varepsilon \in g_{\xi}\left(s_{i}^{\xi}, \nu\right)\left(\left\langle\beta_{\nu, \jmath}^{\xi}, b_{\nu, \jmath}\right\rangle\right)+1 ; s_{i}^{\xi} \Vdash_{T} \text { " } \varepsilon<_{\dot{T}^{b_{\nu, \jmath}}} \beta_{\nu, \jmath}^{\xi} \text { " }\right\} \\
& \quad=\left\{\varepsilon \in g_{\xi}\left(s_{i}^{\xi}, \nu\right)\left(\left\langle\beta_{\nu, \jmath}^{\eta}, b_{\nu, \jmath}\right\rangle\right)+1 ; s_{i}^{\eta} \Vdash_{T} \text { " } \varepsilon<_{\dot{T}^{b_{\nu, \jmath}}} \beta_{\nu, \jmath}^{\eta} \text { " }\right\} .
\end{aligned}
$$

Since $T$ is ccc, by shrinking $\left\{\left\langle\left\langle\sigma_{\xi}, f_{\xi}, g_{\xi}\right\rangle, t_{\xi}\right\rangle ; \xi \in \omega_{1}\right\}$ if necessary, we may assume that there exists $r \in T$ such that $\gamma_{0} \leq \operatorname{lv}(r)<\delta_{0}, r \leq_{T} s_{0}^{\xi}$ for all $\xi \in \omega_{1}$, and the set $\left\{s_{0}^{\xi}\left\lceil\gamma_{\xi} ; \xi \in \omega_{1}\right\}\right.$ is dense above $r$. For each $i \in n$, we write $r_{i}:=\psi_{r, s_{i}^{0} \uparrow \operatorname{lv}(r)}(r)=s_{i}^{0} \uparrow \operatorname{lv}(r)$. Then for all $i \in n$ and $\xi \in \omega_{1}$, $r_{i} \leq_{T} s_{i}^{\xi}$, and the set $\left\{s_{i}^{\xi}\left\lceil\gamma_{\xi} ; \xi \in \omega_{1}\right\}\right.$ is dense above $r_{i}$, because by our assumption,

$$
\psi_{r, s_{i}^{0} \uparrow \operatorname{lv}(r)}\left(s_{0}^{\xi}\left\lceil\gamma_{\xi}\right)=s_{i}^{\xi}\left\lceil\gamma_{\xi}\right.\right.
$$

for all $\xi \in \omega_{1}$ and $i \in n$. By our $\Delta$-system refinement,

$$
\left\{r_{i} ; i \in n\right\}=\left\{r_{j} ; j \in m\right\} .
$$

Note that, by our $\Delta$-system refinement and the definition of the forcing notion $g \mathrm{D}\left(\left\langle\dot{C}_{n} ; n \in \omega\right\rangle, Z\right)$, for any uncountable subsets $I$ and $J$ of $\omega_{1}, \nu \in \sigma$ and $i, j \in n$, if $r_{i}=r_{j}$, then $r_{i} \Vdash_{T}$ "the family

$$
\left\{\left\langle f_{\xi}\left(s_{i}^{\xi}, \nu\right), g_{\xi}\left(s_{i}^{\xi}, \nu\right)\right\rangle ; \xi \in I\right\} \cup\left\{\left\langle f_{\eta}\left(s_{j}^{\eta}, \nu\right), g_{\eta}\left(s_{j}^{\eta}, \nu\right)\right\rangle ; \eta \in J\right\}
$$

forms a suitable refinement in the sense of the proof of Lemma 2.1".

We enumerate the set $\left\{\langle i, j\rangle \in m \times n ; r_{i}=r_{j}\right\}$ as $\left\langle\left\langle i_{\mu}, j_{\mu}\right\rangle ; \mu \in N\right\rangle$ with $\left\langle i_{N-1}, j_{N-1}\right\rangle=\langle 0,0\rangle$. We recursively find $T$-names $\dot{\mathbf{K}}_{\mu}$ and $\dot{\mathbf{L}}_{\mu}$ for uncountable subsets of $\prod_{\nu \in \omega} \mathrm{D}\left(\dot{C}_{\nu}\right)$ below $r_{i_{\mu}}$ and uncountable subsets $I_{\mu}$ and $J_{\mu}$ of $\omega_{1}$ such that for each $\mu \in N$,

- we let $I_{-1}=J_{-1}=\omega_{1}$,

- $r_{i_{\mu}} \Vdash_{T}$ " $\dot{\mathbf{K}}_{\mu} \cap \dot{\mathbf{L}}_{\mu}=\emptyset$ and for any $p \in \dot{\mathbf{K}}_{\mu}$ and $q \in \dot{\mathbf{L}}_{\mu}, p \not \not_{\prod_{\nu \in \omega} \mathrm{D}\left(\dot{C}_{\nu}\right)} q$ ",

- - for any $\xi \in I_{\mu}$ and $u \in T$, if

$$
u \Vdash_{T} \text { " }\left\langle\left\langle f_{\xi}\left(s_{i_{\mu}}^{\xi}, \nu\right), g_{\xi}\left(s_{i_{\mu}}^{\xi}, \nu\right)\right\rangle ; \nu \in \sigma\right\rangle \in \dot{\mathbf{K}}_{\mu} ",
$$

then $s_{i_{\mu}}^{\xi} \leq_{T} u$ and for every $\mu^{\prime}<\mu$,

$$
\psi_{s_{i_{\mu}}^{\xi}, s_{\mu^{\prime}}^{\xi}}(u) \Vdash_{T} \text { “ }\left\langle\left\langle f_{\xi}\left(s_{i_{\mu^{\prime}}}^{\xi}, \nu\right), g_{\xi}\left(s_{i_{\mu^{\prime}}}^{\xi}, \nu\right)\right\rangle ; \nu \in \sigma\right\rangle \in \dot{\mathbf{K}}_{\mu^{\prime}} ",
$$

- for any $\eta \in J_{\mu}$ and $v \in T$, if

$$
v \Vdash_{T} "\left\langle\left\langle f_{\eta}\left(s_{j_{\mu}}^{\eta}, \nu\right), g_{\eta}\left(s_{j_{\mu}}^{\eta}, \nu\right)\right\rangle ; \nu \in \sigma\right\rangle \in \dot{\mathbf{L}}_{\mu} ",
$$

then $s_{j_{\mu}}^{\eta} \leq_{T} v$ and for every $\mu^{\prime}<\mu$,

$$
\psi_{s_{i_{\mu}}^{\eta}, s_{\mu_{\mu^{\prime}}}^{\eta}}(v) \Vdash_{T} \text { “ }\left\langle\left\langle f_{\eta}\left(s_{j_{\mu^{\prime}}}^{\eta}, \nu\right), g_{\eta}\left(s_{j_{\mu^{\prime}}}^{\eta}, \nu\right)\right\rangle ; \nu \in \sigma\right\rangle \in \dot{\mathbf{L}}_{\mu^{\prime}} ",
$$


- we let

$$
\begin{aligned}
& I_{\mu}:=\left\{\xi \in I_{\mu-1} ; \exists u \in T\left(u \Vdash_{T} \text { " }\left\langle\left\langle f_{\xi}\left(s_{i_{\mu}}^{\xi}, \nu\right), g_{\xi}\left(s_{i_{\mu}}^{\xi}, \nu\right)\right\rangle ; \nu \in \sigma\right\rangle \in \dot{\mathbf{K}}_{\mu} "\right)\right\}, \\
& J_{\mu}:=\left\{\eta \in J_{\mu-1} ; \exists u \in T\left(u \Vdash_{T} "\left\langle\left\langle\left\langle f_{\eta}\left(s_{j_{\mu}}^{\eta}, \nu\right), g_{\eta}\left(s_{j_{\mu}}^{\eta}, \nu\right)\right\rangle ; \nu \in \sigma\right\rangle \in \dot{\mathbf{L}}_{\mu} "\right)\right\} .\right.
\end{aligned}
$$

To do this, at stage $\mu \in N$, we define $T$-names ${\underset{\sim}{\mathbf{K}}}_{\mu}$ and $\underset{\sim}{\mathbf{L}} \mu$ such that

- $r_{i_{\mu}} \Vdash_{T}{ }^{\text {" }}{\underset{\sim}{\mu}}_{\mu} \subset\left\{\left\langle\left\langle f_{\xi}\left(s_{i_{\mu}}^{\xi}, \nu\right), g_{\xi}\left(s_{i_{\mu}}^{\xi}, \nu\right)\right\rangle ; \nu \in \sigma\right\rangle ; \xi \in I_{\mu-1}\right\}$ and $\underset{\sim}{\mathbf{L}}{ }_{\mu} \subset\left\{\left\langle\left\langle f_{\eta}\left(s_{j_{\mu}}^{\eta}, \nu\right), g_{\eta}\left(s_{j_{\mu}}^{\eta}, \nu\right)\right\rangle ; \nu \in \sigma\right\rangle ; \eta \in J_{\mu-1}\right\} "$,

- for any $\xi \in I_{\mu-1}$ and $u \in T$,

$$
\begin{aligned}
& u \Vdash_{T} \text { " }\left\langle\left\langle f_{\xi}\left(s_{i_{\mu}}^{\xi}, \nu\right), g_{\xi}\left(s_{i_{\mu}}^{\xi}, \nu\right)\right\rangle ; \nu \in \sigma\right\rangle \in \underset{\sim}{\mathbf{K}_{\mu}} " \\
& : \Leftrightarrow s_{i_{\mu}}^{\xi} \leq_{T} u \& \\
& \quad \forall \mu^{\prime}<\mu\left(\psi_{s_{i_{\mu}}^{\xi}, s_{i_{\mu^{\prime}}}^{\xi}}(u) \Vdash_{T} "\left\langle\left\langle f_{\xi}\left(s_{i_{\mu^{\prime}}}^{\xi}, \nu\right), g_{\xi}\left(s_{i_{\mu^{\prime}}}^{\xi}, \nu\right)\right\rangle ; \nu \in \sigma\right\rangle \in \dot{\mathbf{K}}_{\mu^{\prime}} "\right),
\end{aligned}
$$

and for any $\eta \in J_{\mu-1}$ and $v \in T$,

$v \Vdash_{T}$ " $\left\langle\left\langle f_{\eta}\left(s_{j_{\mu}}^{\eta}, \nu\right), g_{\eta}\left(s_{j_{\mu}}^{\eta}, \nu\right)\right\rangle ; \nu \in \sigma\right\rangle \in \underset{\sim}{\mathbf{L}} \mu$

$: \Leftrightarrow s_{j_{\mu}}^{\eta} \leq_{T} v \&$

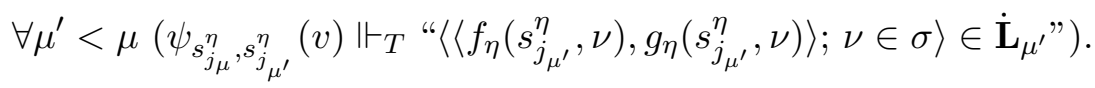

We notice that

$r_{i_{\mu}} \Vdash_{T}$ "both $\underset{\sim}{\mathbf{K}_{\mu}}$ and $\underset{\sim}{\mathbf{L}}$ are uncountable subsets of $\prod_{\nu \in \omega} \mathrm{D}\left(\dot{C}_{\nu}\right)$

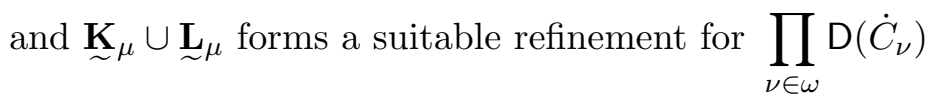

in the sense of the proof of Lemma 2.1".

So there are $T$-names $\dot{\mathbf{K}}_{\mu}$ and $\dot{\mathbf{L}}_{\mu}$ for uncountable subsets of $\underset{\sim}{\mathbf{K}}$ and $\underset{\sim}{\mathbf{L}_{\mu}}$ respectively such that

$$
r_{i_{\mu}} \Vdash_{T} \text { " } \dot{\mathbf{K}}_{\mu} \cap \dot{\mathbf{L}}_{\mu}=\emptyset \text { and for any } p \in \dot{\mathbf{K}}_{\mu} \text { and } q \in \dot{\mathbf{L}}_{\mu}, p \not \not_{\prod_{\nu \in \omega} \mathrm{D}\left(\dot{C}_{\nu}\right)} q " \text {. }
$$

Then we notice that $I_{\mu}$ and $J_{\mu}$, defined as above, are both uncountable, and this finishes the constructions.

We take some (or any) $\xi \in I_{N-1}$. Then there exists $u \in T$ such that

$$
u \Vdash_{T} \text { " }\left\langle\left\langle f_{\xi}\left(s_{i_{N-1}}^{\xi}, \nu\right), g_{\xi}\left(s_{i_{N-1}}^{\xi}, \nu\right)\right\rangle ; \nu \in \sigma\right\rangle \in \dot{\mathbf{K}}_{N-1} " .
$$

Then by our constructions, $s_{i_{N-1}}^{\xi} \leq_{T} u$ and for any $\mu \in N$,

$$
\psi_{s_{i_{N-1}}^{\xi}, s_{i_{\mu}}^{\xi}}(u) \Vdash_{T} \text { " }\left\langle\left\langle f_{\xi}\left(s_{i_{\mu}}^{\xi}, \nu\right), g_{\xi}\left(s_{i_{\mu}}^{\xi}, \nu\right)\right\rangle ; \nu \in \sigma\right\rangle \in \dot{\mathbf{K}}_{\mu} " .
$$

Since $\dot{\mathbf{L}}_{N-1}$ is forced to be uncountable, there exist $\eta \in \omega_{1}$ and $v \in T$ such 
that $\xi<\eta,\left(\delta_{\xi} \leq\right) \operatorname{lv}(u)<\gamma_{\eta}, u<_{T} v, \delta_{\xi}<\operatorname{lv}(v)$, and

$$
v \Vdash_{T} \text { " }\left\langle\left\langle f_{\eta}\left(s_{j_{N-1}}^{\eta}, \nu\right), g_{\eta}\left(s_{j_{N-1}}^{\eta}, \nu\right)\right\rangle ; \nu \in \sigma\right\rangle \in \dot{\mathbf{L}}_{N-1} " .
$$

(In fact, then $\eta \in J_{N-1}$.) By our constructions, $s_{j_{N-1}}^{\eta} \leq_{T} v$, and for any $\mu \in N$,

$$
\psi_{s_{j_{N-1}}^{\eta}, s_{j_{\mu}}^{\eta}}(v) \Vdash_{T} "\left\langle\left\langle f_{\eta}\left(s_{j_{\mu}}^{\eta}, \nu\right), g_{\eta}\left(s_{j_{\mu}}^{\eta}, \nu\right)\right\rangle ; \nu \in \sigma\right\rangle \in \dot{\mathbf{L}}_{\mu} " .
$$

We notice that, since

$$
t_{\xi}=s_{0}^{\xi}=s_{i_{N-1}}^{\xi} \leq_{T} u<_{T} v, \quad t_{\eta}=s_{0}^{\eta}=s_{j_{N-1}}^{\eta} \leq_{T} v
$$

and $\operatorname{lv}\left(t_{\xi}\right)=\delta_{\xi} \leq \operatorname{Iv}(u)<\delta_{\eta}=\operatorname{Iv}\left(t_{\eta}\right)$, it follows that $t_{\xi} \leq_{T} u<_{T} t_{\eta}$. Therefore by our $\Delta$-system refinement and $\delta_{\xi} \leq \operatorname{lv}(u)<\gamma_{\eta}$, for each $w \in$ $\operatorname{dom}\left(f_{\eta}\right) \backslash R$, we can find $\mu_{w} \in N$ such that $w<_{T} s_{j_{\mu_{w}}}^{\eta}$ and $s_{i_{\mu_{w}}}^{\xi}<_{T} s_{j_{\mu_{w}}}^{\eta}$. We then notice that for every $w \in \operatorname{dom}\left(f_{\eta}\right) \backslash R$, since

$$
\begin{gathered}
s_{i_{\mu_{w}}}^{\xi}=r_{i_{\mu_{w}}} \frown t_{\xi} \uparrow\left[\operatorname{lv}(r), \delta_{\xi}\right) \leq_{T} r_{i_{\mu_{w}}} \frown t_{\eta}\left\lceil[\operatorname{lv}(r), \operatorname{lv}(u))=\psi_{s_{i_{N-1}}^{\xi}, s_{i_{\mu_{w}}}^{\xi}}(u)\right. \\
<_{T} r_{i_{\mu_{w}}} \frown t_{\eta} \uparrow[\operatorname{lv}(r), \operatorname{lv}(w))=w<_{T} s_{j_{\mu_{w}}}^{\eta} \leq_{T} \psi_{s_{j_{N-1}}^{\eta}, s_{j_{\mu_{w}}}^{\eta}(v),}
\end{gathered}
$$

it follows that

$$
\begin{aligned}
\psi_{s_{j_{N-1}}^{\eta}}, s_{j_{\mu_{w}}}^{\eta}(v) \Vdash_{T} "\left\langle\left\langle f_{\xi}\left(s_{i_{\mu_{w}}}^{\xi}, \nu\right), g_{\xi}\left(s_{i_{\mu_{w}}}^{\xi}, \nu\right)\right\rangle ; \nu \in \sigma\right\rangle \in \dot{\mathbf{K}}_{\mu_{w}} \\
\quad \text { and }\left\langle\left\langle f_{\eta}\left(s_{j_{\mu_{w}}}^{\eta}, \nu\right), g_{\eta}\left(s_{j_{\mu_{w}}}^{\eta}, \nu\right)\right\rangle ; \nu \in \sigma\right\rangle \in \dot{\mathbf{L}}_{\mu_{w}} ",
\end{aligned}
$$

hence

$$
\begin{aligned}
w \Vdash_{T} "\left\langle\left\langle f_{\xi}\left(s_{i_{\mu_{w}}}^{\xi}, \nu\right), g_{\xi}\left(s_{i_{\mu_{w}}}^{\xi}, \nu\right)\right\rangle: \nu \in \sigma\right\rangle & \text { and }\left\langle\left\langle f_{\eta}(w, \nu), g_{\eta}(w, \nu)\right\rangle ; \nu \in \sigma\right\rangle \text { are compatible". }
\end{aligned}
$$

Let

$$
\begin{aligned}
& f:=f_{\xi} \cup\left\{\left\langle w,\left\langle f_{\xi}\left(s_{i_{\mu_{w}}}^{\xi}, \nu\right) \cup f_{\eta}(w, \nu) ; \nu \in \sigma\right\rangle\right\rangle ; w \in \operatorname{dom}\left(f_{\eta}\right) \backslash R\right\}, \\
& g:=g_{\xi} \cup\left\{\left\langle w,\left\langle g_{\xi}\left(s_{i_{\mu_{w}}}^{\xi}, \nu\right) \cup g_{\eta}(w, \nu) ; \nu \in \sigma\right\rangle\right\rangle ; w \in \operatorname{dom}\left(f_{\eta}\right) \backslash R\right\} .
\end{aligned}
$$

Then $\left\langle\langle\sigma, f, g\rangle, t_{\eta}\right\rangle$ is a condition of $g \mathrm{D}\left(\left\langle\dot{C}_{\nu} ; \nu \in \omega\right\rangle, Z\right)$ and is a common extension of $\left\langle\left\langle\sigma_{\xi}, f_{\xi}, g_{\xi}\right\rangle, t_{\xi}\right\rangle$ and $\left\langle\left\langle\sigma_{\eta}, f_{\eta}, g_{\eta}\right\rangle, t_{\eta}\right\rangle$.

Therefore by the standard argument to force a fragment of forcing axioms (e.g. $[4, \S 3],[5, \S 4]$ or $[6, \S 8]$ ), we can deduce Theorem 3(1). For example, under the existence of a coherent Suslin tree $T$, we just force by iterating, with finite supports, the forcing notions $\mathbb{P}$ of size $\aleph_{1}$ such that $\mathbb{P} \times T$ is ccc. This is discussed in [12, Theorem 4.6].

3.2. Proof of Theorem 3(2). Throughout this subsection, we suppose that $\left\langle\dot{T}^{n} ; n \in \omega\right\rangle$ is a sequence of $T$-names for Aronszajn trees and $\left\langle\dot{\pi}_{n}\right.$; $n \in \omega \backslash\{0\}\rangle$ is a sequence of $T$-names for functions with (p1-4) such that 
it is forced that for each $n \in \omega$ and each $\alpha \in \omega_{1}$, the set $\left(\dot{T}^{n}\right)_{\alpha}$ is a subset of the interval $\left[\lambda_{\alpha}, \lambda_{\alpha}+\omega\right)$.

Let $\dot{C}$ and $\dot{D}$ be $T$-names for closed subsets of $\mathfrak{X}\left(\left\langle\dot{T}^{n}, \dot{\pi}^{n} ; n \in \omega\right\rangle\right)$ such that it is forced that $\dot{C}$ and $\dot{D}$ are disjoint. As in the previous subsection, we can find a club $Z$ on $\omega_{1}$ such that $\lambda_{\gamma}=\gamma$ for every $\gamma \in Z$, and for any $\alpha, \beta \in \gamma$ and $m, n \in \omega$, every node in $T_{\gamma}$ decides the statements " $\alpha \in \dot{T}^{n}$ ", " $\alpha \leq_{\dot{T}^{n}} \beta ", "\langle\alpha, \beta\rangle \in \dot{\pi}^{n "}, "\langle\alpha, m\rangle \in \dot{C} "$, and " $\langle\alpha, m\rangle \in \dot{D} "$.

As above, we define a forcing notion

$$
g_{T} \mathrm{~N}\left(\left\langle\dot{T}^{n}, \dot{\pi}^{n} ; n \in \omega\right\rangle, \dot{C}, \dot{D}, Z\right),
$$

or $g \mathrm{~N}(\dot{C}, \dot{D}, Z)$ for short, which consists of the pairs $\langle f, g\rangle$ of functions such that

1. $\operatorname{dom}(f)=\operatorname{dom}(g)$ and $\operatorname{dom}(f)$ is a finite subset of $T$ closed under $\wedge$,

2. for each $t \in \operatorname{dom}(f), f(t)$ and $g(t)$ are finite partial functions from $\sup (Z \cap(\operatorname{lv}(t)+1)) \times \omega$ into $\omega_{1}$ and

$$
t \Vdash_{T} \text { “ }\langle f(t), g(t)\rangle \in \mathrm{N}\left(\left\langle\dot{T}^{n}, \dot{\pi}^{n} ; n \in \omega\right\rangle, \dot{C}, \dot{D}\right) ",
$$

3. for all $t, t^{\prime} \in \operatorname{dom}(f)$, if $t^{\prime}<_{T} t$, then

$$
t \Vdash_{T} "\langle f(t), g(t)\rangle \leq_{\mathrm{N}(\dot{C}, \dot{D})}\left\langle f\left(t^{\prime}\right), g\left(t^{\prime}\right)\right\rangle "
$$

and if

$$
(\operatorname{dom}(f(t)) \cup \operatorname{dom}(g(t))) \backslash\left(\operatorname{dom}\left(f\left(t^{\prime}\right)\right) \cup \operatorname{dom}\left(g\left(t^{\prime}\right)\right)\right) \neq \emptyset,
$$

then

$$
\begin{aligned}
& \max \left\{\operatorname{lv}(\alpha) ;\langle\alpha, m\rangle \in \operatorname{dom}\left(f\left(t^{\prime}\right)\right) \cup \operatorname{dom}\left(g\left(t^{\prime}\right)\right) \text { for some } m \in \omega\right\} \\
& <\min \{\operatorname{lv}(\alpha) ; \\
& \langle\alpha, m\rangle \in(\operatorname{dom}(f(t)) \cup \operatorname{dom}(g(t))) \backslash\left(\operatorname{dom}\left(f\left(t^{\prime}\right)\right) \cup \operatorname{dom}\left(g\left(t^{\prime}\right)\right)\right) \\
& \text { for some } m \in \omega\},
\end{aligned}
$$

4. for all $s$ and $t$ in $\operatorname{dom}(f)$,

$$
\begin{aligned}
& \operatorname{dom}(f(s \wedge t)) \\
& \quad=\operatorname{dom}(f(s)) \cap \operatorname{dom}(f(t)) \cap(\sup (Z \cap(\Delta(s, t)+1)) \times \omega), \\
& \operatorname{dom}(g(s \wedge t)) \\
& \quad=\operatorname{dom}(g(s)) \cap \operatorname{dom}(g(t)) \cap(\sup (Z \cap(\Delta(s, t)+1)) \times \omega),
\end{aligned}
$$

5. for all $s$ and $t$ in $\operatorname{dom}(f)$,

$$
\begin{aligned}
(M(f(s), s, \Delta(s, t)) \cup M(f(t), t, \Delta(s, t))) & \\
\cap(M(g(s), s, \Delta(s, t)) \cup M(g(t), t, \Delta(s, t))) & =\emptyset .
\end{aligned}
$$

For conditions $\langle f, g\rangle$ and $\left\langle f^{\prime}, g^{\prime}\right\rangle$ in $g \mathrm{~N}(\dot{C}, \dot{D}, Z)$, we define 


$$
\begin{aligned}
\langle f, g\rangle \leq_{g \mathrm{~N}(\dot{C}, \dot{D}, Z)}\left\langle f^{\prime}, g^{\prime}\right\rangle & \\
\quad \Leftrightarrow & \operatorname{dom}(f) \supseteq \operatorname{dom}\left(f^{\prime}\right) \\
& \quad \& \forall t \in \operatorname{dom}\left(f^{\prime}\right)\left(t \Vdash_{T} "\langle f(t), g(t)\rangle \leq_{\mathrm{N}(\dot{C}, \dot{D})}\left\langle f^{\prime}(t), g^{\prime}(t)\right\rangle "\right) .
\end{aligned}
$$

Proposition 3.5. For each $t \in T$, the set

$$
\left\{\langle f, g\rangle \in g_{T} \mathrm{~N}\left(\left\langle\dot{T}^{n}, \dot{\pi}^{n} ; n \in \omega\right\rangle, \dot{C}, \dot{D}, Z\right) ; t \in \operatorname{dom}(f)\right\}
$$

is dense.

Proof. Let $\langle f, g\rangle \in g \mathrm{~N}(\dot{C}, \dot{D}, Z)$.

If there is $s \in \operatorname{dom}(f)$ so that $t \leq_{T} s$, then

$$
\begin{aligned}
\langle f \cup\{\langle t, f(s)\lceil(\operatorname{dom}(f(s)) & \cap(\sup (Z \cap(\operatorname{lv}(t)+1)) \times \omega))\rangle\}, \\
g & \cup\{\langle t, g(s)\lceil(\operatorname{dom}(g(s)) \cap(\sup (Z \cap(\operatorname{lv}(t)+1)) \times \omega))\rangle\}\rangle
\end{aligned}
$$

is a condition of $g \mathrm{~N}(\dot{C}, \dot{D}, Z)$ and an extension of $\langle f, g\rangle$.

Suppose that $t \not_{T} s$ for all $s \in \operatorname{dom}(f)$. Then there exists $s \in \operatorname{dom}(f)$ such that $\Delta(t, u) \leq \Delta(t, s)$ for any $u \in \operatorname{dom}(f)$. Let

$$
\begin{aligned}
f^{\prime}:= & f \cup\{\langle s \wedge t, f(s)\lceil(\operatorname{dom}(f(s)) \cap(\sup (Z \cap(\Delta(t, s)+1)) \times \omega))\rangle\} \\
& \cup\{\langle t, f(s)\lceil(\operatorname{dom}(f(s)) \cap(\sup (Z \cap(\Delta(t, s)+1)) \times \omega))\rangle\}, \\
g^{\prime}:= & g \cup\{\langle s \wedge t, g(s)\lceil(\operatorname{dom}(g(s)) \cap(\sup (Z \cap(\Delta(t, s)+1)) \times \omega))\rangle\} \\
& \cup\{\langle t, g(s)\lceil(\operatorname{dom}(g(s)) \cap(\sup (Z \cap(\Delta(t, s)+1)) \times \omega))\rangle\} .
\end{aligned}
$$

Then $\left\langle f^{\prime}, g^{\prime}\right\rangle$ is a condition of $g \mathrm{~N}(\dot{C}, \dot{D}, Z)$ and an extension of $\langle f, g\rangle$.

Proposition 3.6. For $t \in T$ and $\langle\alpha, m\rangle \in \omega_{1} \times \omega$, the set

$$
\begin{aligned}
\left\{\langle f, g\rangle \in g_{T} \mathrm{~N}\left(\left\langle\dot{T}^{n}, \dot{\pi}^{n} ; n \in \omega\right\rangle, \dot{C}, \dot{D}, Z\right) ; \exists s \in \operatorname{dom}(f)\left(t \leq_{T} s \&\right.\right. \\
\left.\left.\left(\text { either } s \Vdash_{T} \text { " } \alpha \notin \dot{T}^{m} " \text { or }\langle\alpha, m\rangle \in \operatorname{dom}(f(s)) \cup \operatorname{dom}(g(s))\right)\right)\right\}
\end{aligned}
$$

is dense.

Proof. Let $\langle f, g\rangle \in g \mathrm{~N}(\dot{C}, \dot{D}, Z)$. From the previous proposition, we may assume that $t \in \operatorname{dom}(f)$ and there exists $s \in T$ such that $t \leq_{T} s, \operatorname{lv}(u) \leq$ $\operatorname{lv}(s)$ for every $u \in \operatorname{dom}(f)$, and $\operatorname{lv}(\alpha)<\sup (Z \cap(\operatorname{lv}(s)+1))$. By the property of $Z, s$ decides the statement " $\alpha \in \dot{T}^{m}$ ". If $s \Vdash_{T}$ " $\alpha \notin \dot{T}^{m}$ ", then $\langle f, g\rangle$ is in the above set, so now we suppose that $s \Vdash$ " $\alpha \in \dot{T}^{m}$ ".

We enumerate the set

$$
\{u \in \operatorname{dom}(f) ; \operatorname{lv}(\alpha) \in \sup (Z \cap(\Delta(u, s)+1))\}
$$

as $\left\langle u_{i} ; i \in k\right\rangle$. We note that for any $j \in k$ and $v \in \operatorname{dom}(f), \operatorname{if} \operatorname{lv}(\alpha) \in$ $\sup \left(Z \cap\left(\Delta\left(u_{j}, v\right)+1\right)\right)$, then $v$ is also in $\left\{u_{i} ; i \in k\right\}$, and $v \Vdash_{T} " \alpha \in \dot{T}^{m}$ ". If for some $i \in k,\langle\alpha, m\rangle \in \operatorname{dom}\left(f\left(u_{i}\right)\right) \cup \operatorname{dom}\left(g\left(u_{i}\right)\right)$, then $\langle f, g\rangle$ is in the above set, so suppose that $\langle\alpha, m\rangle \notin \operatorname{dom}\left(f\left(u_{i}\right)\right) \cup \operatorname{dom}\left(g\left(u_{i}\right)\right)$ for any $i \in k$. By the definition of $g \mathrm{~N}(\dot{C}, \dot{D}, Z)$, one of the following happens: 
- For all $i, j \in k,\langle\alpha, m\rangle \notin M\left(f\left(u_{i}\right), u_{i}, \Delta\left(u_{i}, u_{j}\right)\right)$.

- For all $i, j \in k,\langle\alpha, m\rangle \notin M\left(g\left(u_{i}\right), u_{i}, \Delta\left(u_{i}, u_{j}\right)\right)$.

Without loss of generality, we may assume that the first case happens. Then we recursively pick $\gamma_{i} \in \operatorname{lv}(\alpha)$ for $i \in k$ such that:

- for each $i \in k-1, \gamma_{i} \leq \gamma_{i+1}$,

- for each $i \in k$,

$$
u_{i} \Vdash_{T} \text { " }\left\langle f\left(u_{i}\right), g\left(u_{i}\right) \cup\left\{\left\langle\langle\alpha, m\rangle, \gamma_{i}\right\rangle\right\}\right\rangle \in \mathrm{N}(\dot{C}, \dot{D}) ",
$$

- for all $i \in k$ and $v \in \operatorname{dom}(f)$,

$$
M\left(f(v), v, \Delta\left(u_{i}, v\right)\right) \cap M\left(g\left(u_{i}\right) \cup\left\{\left\langle\langle\alpha, m\rangle, \gamma_{i}\right\rangle\right\}, u_{i}, \Delta\left(u_{i}, v\right)\right)=\emptyset .
$$

Then the pair

$$
\left\langle f,\left(g \uparrow\left(\operatorname{dom}(g) \backslash\left\{u_{i} ; i \in k\right\}\right)\right) \cup\left\{\left\langle u_{i}, g\left(u_{i}\right) \cup\left\{\left\langle\langle\alpha, m\rangle, \gamma_{k-1}\right\rangle\right\}\right\rangle ; i \in k\right\}\right\rangle
$$

is a condition of $g \mathrm{~N}(\dot{C}, \dot{D}, Z)$ and an extension of $\langle f, g\rangle$.

Assume that $g \mathrm{~N}(\dot{C}, \dot{D}, Z) \times T$ is ccc. (Then so is $g \mathrm{~N}(\dot{C}, \dot{D}, Z)$.) We denote the ground model by $\mathbf{V}$ and let $G$ be a $g \mathrm{~N}(\dot{C}, \dot{D}, Z)$-generic filter over $\mathbf{V}$, and $H$ a $T$-generic filter over $\mathbf{V}[G]$. Set

$$
\begin{aligned}
G_{H}:=\{\langle c, d\rangle \in \mathrm{N}(\dot{C}[H], \dot{D}[H]) ; & \exists\langle f, g\rangle \in G \exists t \in \operatorname{dom}(f) \cap H \\
& \left.\left(t \Vdash_{T} "\langle f(t), g(t)\rangle \leq_{\mathrm{N}(\dot{C}, \dot{D})}\langle c, d\rangle "\right)\right\} .
\end{aligned}
$$

$(\dot{C}[H]$ and $\dot{D}[H]$ are the interpretations of $\dot{C}$ and $\dot{D}$ respectively in $\mathbf{V}[G][H]$.) By the definition of $g \mathrm{~N}(\dot{C}, \dot{D}, Z), G_{H}$ is a filter, and by the previous proposition, we note that in $\mathbf{V}[G]$, for any $\langle\alpha, m\rangle \in \omega_{1} \times \omega$, the set

$\left\{s \in T\right.$; either $s \Vdash_{T}$ " $\alpha \notin \dot{T}^{m}$ " or $\left.\langle\alpha, m\rangle \in \operatorname{dom}(f(s)) \cup \operatorname{dom}(g(s))\right\}$

is dense in $T$, so $G_{H}$ generates disjoint open sets which separate $\dot{C}[G]$ and $\dot{D}[G]$.

LEMma 3.7. $g_{T} \mathrm{~N}\left(\left\langle\dot{T}^{n}, \dot{\pi}^{n} ; n \in \omega\right\rangle, \dot{C}, \dot{D}, Z\right) \times T$ satisfies the countable chain condition.

Proof. Let $\left\{\left\langle\left\langle f_{\xi}, g_{\xi}\right\rangle, t_{\xi}\right\rangle ; \xi \in \omega_{1}\right\}$ be an uncountable subset of $g \mathrm{~N}(\dot{C}, \dot{D}, Z)$ $\times T$. By strengthening each condition if necessary, we may assume that for each $\xi \in \omega_{1}$, there is $\delta_{\xi}$ in $\omega_{1}$ such that

- $t_{\xi} \in \operatorname{dom}\left(f_{\xi}\right) \cap T_{\delta_{\xi}}$ and every node in $\operatorname{dom}\left(f_{\xi}\right)$ is of height $\leq \delta_{\xi}$,

- for any $t \in \operatorname{dom}\left(f_{\xi}\right)$, there exists $t^{\prime} \in \operatorname{dom}\left(f_{\xi}\right) \cap T_{\delta_{\xi}}$ such that $t \leq_{T} t^{\prime}$. By shrinking the subset and strengthening each condition again if necessary, we may also assume that there exists $\gamma_{0} \in \omega_{1}$ such that

- $\gamma_{0}<\delta_{0}$ and if $\xi<\eta$ in $\omega_{1}$, then $\delta_{\xi}<\delta_{\eta}$,

- $\left\{\operatorname{dom}\left(f_{\xi}\right) ; \xi \in \omega_{1}\right\}$ is a $\Delta$-system with a root $R$ such that $R \subseteq T_{\leq \gamma_{0}}$ and if $\xi<\eta$ in $\omega_{1}$, then $f_{\xi}\left\lceil R=f_{\eta}\left\lceil R\right.\right.$ and $\delta_{\xi}<\operatorname{lv}(s)$ for any $s \in \operatorname{dom}\left(f_{\eta}\right) \backslash R$, 
- - $\left\{\bigcup_{t \in \operatorname{dom}\left(f_{\xi}\right)} \operatorname{dom}\left(f_{\xi}(t)\right) ; \xi \in \omega_{1}\right\}$ is a $\Delta$-system with a root $S_{0} \subseteq$ $\gamma_{0} \times \omega$, and if $\xi<\eta$ in $\omega_{1}$, then $\delta_{\xi}<\operatorname{lv}(\varepsilon)$ for any $\langle\varepsilon, m\rangle \in$ $\bigcup_{t \in \operatorname{dom}\left(f_{\eta}\right)} \operatorname{dom}\left(f_{\eta}(t)\right) \backslash S_{0}$,

- $\left\{\bigcup_{t \in \operatorname{dom}\left(g_{\xi}\right)} \operatorname{dom}\left(g_{\xi}(t)\right) ; \xi \in \omega_{1}\right\}$ is a $\Delta$-system with a root $S_{1} \subseteq$ $\gamma_{0} \times \omega$, and if $\xi<\eta$ in $\omega_{1}$, then $\delta_{\xi}<\operatorname{lv}(\varepsilon)$ for any $\langle\varepsilon, m\rangle \in$ $\bigcup_{t \in \operatorname{dom}\left(g_{\eta}\right)} \operatorname{dom}\left(g_{\eta}(t)\right) \backslash S_{1}$,

- there is a strictly increasing sequence $\left\langle\gamma_{\xi} ; \xi \in \omega_{1} \backslash\{0\}\right\rangle$ of countable ordinals such that for every $\xi \in \omega_{1}$,

$$
\left\{\zeta \in \omega_{1} ; \exists s, t \in \operatorname{dom}\left(f_{\xi}\right) \cap T_{\delta_{\xi}}\left(\zeta \in \delta_{\xi} \& s(\zeta) \neq t(\zeta)\right)\right\}
$$

is a subset of $\gamma_{0} \cup\left[\gamma_{\xi}, \gamma_{\xi+1}\right)$,

- $\left|\operatorname{dom}\left(f_{\xi}\right) \cap T_{\delta_{\xi}}\right|=: n$ for all $\xi \in \omega_{1}$, say

$$
\operatorname{dom}\left(f_{\xi}\right) \cap T_{\delta_{\xi}}=\left\{s_{0}^{\xi}, s_{1}^{\xi}, \ldots, s_{n-1}^{\xi}\right\},
$$

and $t_{\xi}=s_{0}^{\xi}$, and there exists $m \leq n$ such that for any $\xi \in \omega_{1}$ and $i \in n$, there is a unique $j \in m$ such that $s_{i}^{\xi}\left\lceil\gamma_{\xi}=s_{j}^{\xi}\left\lceil\gamma_{\xi}\right.\right.$ and $s_{j}^{\xi}\left\lceil\left[\gamma_{\xi}, \gamma_{\xi+1}\right)=\right.$ $t_{\xi} \uparrow\left[\gamma_{\xi}, \gamma_{\xi+1}\right)$, and for all $i \in n$ and $\xi, \eta \in \omega_{1}, s_{i}^{\xi}\left\lceil\gamma_{0}=s_{i}^{\eta}\left\lceil\gamma_{0}\right.\right.$,

- for any $\xi, \eta \in \omega_{1}$ and $i \in n, f_{\xi}\left(s_{i}^{\xi}\right) \mid S_{0}=f_{\eta}\left(s_{i}^{\eta}\right)\left\lceil S_{0}\right.$ and $g_{\xi}\left(s_{i}^{\xi}\right)\left\lceil S_{1}=\right.$ $g_{\eta}\left(s_{i}^{\eta}\right)\left\lceil S_{1}\right.$,

- for each $i \in n$,

- the size of all $\operatorname{dom}\left(f_{\xi}\left(s_{i}^{\xi}\right)\right) \backslash S_{0}$ is the same, say $k_{i}$, the size of all $\operatorname{dom}\left(g_{\xi}\left(s_{i}^{\xi}\right)\right) \backslash S_{1}$ is the same, say $l_{i}$, and $\operatorname{dom}\left(f_{\xi}\left(s_{i}^{\xi}\right)\right) \backslash S_{0}=$ $\left\{\left\langle\alpha_{\imath}^{\xi}, a_{\imath}\right\rangle ; \imath \in k_{i}\right\}$ and $\operatorname{dom}\left(g_{\xi}\left(s_{i}^{\xi}\right)\right) \backslash S_{1}=\left\{\left\langle\beta_{\jmath}^{\xi}, b_{\jmath}\right\rangle ; \jmath \in l_{i}\right\}$ for some $a_{\imath}$ and $b_{\jmath}$,

- there are $K_{i} \subseteq k_{i}$ and $L_{i} \subseteq l_{i}$ such that

$*$ if $\xi<\eta$ in $\omega_{1}$ and $\imath \in k_{i} \backslash K_{i}$ and $\jmath \in l_{i} \backslash L_{i}$, then

$$
\begin{gathered}
\delta_{\xi}<f_{\eta}\left(s_{i}^{\eta}\right)\left(\left\langle\alpha_{\imath}^{\eta}, a_{\imath}\right\rangle\right) \quad \text { and } \quad \delta_{\xi}<g_{\eta}\left(s_{i}^{\eta}\right)\left(\left\langle\beta_{\jmath}^{\eta}, b_{\jmath}\right\rangle\right), \\
\left\{\varepsilon \in \gamma_{0}+1 ; s_{i}^{\xi} \Vdash_{T} " \varepsilon<_{\dot{T}^{a_{\imath}}} \alpha_{\imath}^{\xi "}\right\} \\
=\left\{\varepsilon \in \gamma_{0}+1 ; s_{i}^{\eta} \Vdash_{T} \text { “ } \varepsilon<_{\dot{T}^{a_{\imath}}} \alpha_{\imath}^{\eta ”}\right\}
\end{gathered}
$$

and

$$
\begin{aligned}
\left\{\varepsilon \in \gamma_{0}+1 ; s_{i}^{\xi} \Vdash_{T} " \varepsilon\right. & \left.<_{\dot{T}^{b_{j}}} \beta_{j}^{\xi "}\right\} \\
& =\left\{\varepsilon \in \gamma_{0}+1 ; s_{i}^{\eta} \Vdash_{T} " \varepsilon<_{\dot{T}^{b_{j}}} \beta_{j}^{\eta ”}\right\},
\end{aligned}
$$

$*$ if $\xi, \eta \in \omega_{1}$ and $\imath \in K_{i}$ and $\jmath \in L_{i}$, then

$$
\begin{aligned}
& f_{\xi}\left(s_{i}^{\xi}\right)\left(\left\langle\alpha_{\imath}^{\xi}, a_{\imath}\right\rangle\right)=f_{\eta}\left(s_{i}^{\eta}\right)\left(\left\langle\alpha_{\imath}^{\eta}, a_{\imath}\right\rangle\right), \\
& g_{\xi}\left(s_{i}^{\xi}\right)\left(\left\langle\beta_{\jmath}^{\xi}, b_{\jmath}\right\rangle\right)=g_{\eta}\left(s_{i}^{\eta}\right)\left(\left\langle\beta_{\jmath}^{\eta}, b_{\jmath}\right\rangle\right),
\end{aligned}
$$




$$
\begin{aligned}
\left\{\varepsilon \in f_{\xi}\left(s_{i}^{\xi}\right)\left(\alpha_{\imath}^{\xi}\right)\right. & \left.+1 ; s_{i}^{\xi} \Vdash_{T} " \varepsilon<_{\dot{T}^{a_{\imath}}} \alpha_{\imath}^{\xi "}\right\} \\
& =\left\{\varepsilon \in f_{\xi}\left(s_{i}^{\xi}\right)\left(\alpha_{\imath}^{\xi}\right)+1 ; s_{i}^{\eta} \Vdash_{T}{ }^{~ " \varepsilon} \varepsilon<_{\dot{T}^{a_{\imath}}} \alpha_{\imath}^{\eta "}\right\}
\end{aligned}
$$

and

$$
\begin{aligned}
\left\{\varepsilon \in g_{\xi}\left(s_{i}^{\xi}\right)\left(\beta_{\jmath}^{\xi}\right)\right. & \left.+1 ; s_{i}^{\xi} \Vdash_{T} " \varepsilon<_{\dot{T}^{b_{\jmath}}} \beta_{\jmath}^{\xi "}\right\} \\
& =\left\{\varepsilon \in g_{\xi}\left(s_{i}^{\xi}\right)\left(\beta_{\jmath}^{\xi}\right)+1 ; s_{i}^{\eta} \Vdash_{T} " \varepsilon<_{\dot{T}^{b_{\jmath}}} \beta_{\jmath}^{\eta "}\right\} .
\end{aligned}
$$

Since $T$ is ccc, as in the proof of Lemma 3.4, by shrinking the family $\left\{\left\langle\left\langle f_{\xi}, g_{\xi}\right\rangle, t_{\xi}\right\rangle ; \xi \in \omega_{1}\right\}$ if necessary, we may assume that there exists $r \in T$ such that $\gamma_{0} \leq \operatorname{lv}(r)<\delta_{0}$ and $r \leq_{T} s_{0}^{\xi}$ for all $\xi \in \omega_{1}$, and the set $\left\{s_{0}^{\xi}\left\lceil\gamma_{\xi} ; \xi \in \omega_{1}\right\}\right.$ is dense above $r$. For each $i \in n$, we write $r_{i}:=$ $\psi_{r, s_{i}^{0} \uparrow \operatorname{lv}(r)}(r)=s_{i}^{0} \uparrow \operatorname{lv}(r)$. Then by our $\Delta$-system refinement and the definition of $g \mathrm{~N}(\dot{C}, \dot{D}, Z)$, for any uncountable subsets $I$ and $J$ of $\omega_{1}$ and $i, j \in n$, if $r_{i}=r_{j}$, then $r_{i} \Vdash_{T}$ "the family

$$
\left\{\left\langle f_{\xi}\left(s_{i}^{\xi}\right), g_{\xi}\left(s_{i}^{\xi}\right)\right\rangle ; \xi \in I\right\} \cup\left\{\left\langle f_{\eta}\left(s_{j}^{\eta}\right), g_{\eta}\left(s_{j}^{\eta}\right)\right\rangle ; \eta \in J\right\}
$$

forms a suitable refinement in the sense of the proof of Lemma 2.2".

Therefore the rest of the proof is completely the same as the proof of Lemma 3.4.

4. Generalized Rudin spaces constructed with non-Aronszajn $\omega_{1}$-trees. In this section, we consider generalized Rudin spaces with nonAronszajn $\omega_{1}$-trees. Note that in general, for a decreasing sequence $\left\langle T^{n}\right.$; $n \in \omega\rangle$ of $\omega_{1}$-trees and a sequence $\left\langle\pi^{n} ; n \in \omega \backslash\{0\}\right\rangle$ of functions with (p1-3), and a closed subset $C$ of $\mathfrak{X}\left(\left\langle T^{n}, \pi^{n} ; n \in \omega\right\rangle\right)$, the forcing notion $\mathrm{D}\left(\left\langle T^{n}, \pi^{n}\right.\right.$; $n \in \omega\rangle, C)$ does not satisfy the countable chain condition.

Proposition 4.1. Suppose that $\left\langle T^{n} ; n \in \omega\right\rangle$ is a decreasing sequence of $\omega_{1}$-trees and $\left\langle\pi^{n} ; n \in \omega \backslash\{0\}\right\rangle$ is a sequence of functions with (p1-3) such that $T^{0}$ has a cofinal chain (a cofinal branch). Then there exists a closed subset $C$ of $\mathfrak{X}\left(\left\langle T^{n}, \pi^{n} ; n \in \omega\right\rangle\right)$ such that $\mathrm{D}\left(\left\langle T^{n}, \pi^{n} ; n \in \omega\right\rangle, C\right)$ has an uncountable antichain.

Proof. Let $B$ be a cofinal chain in $T^{0}$, and let

$$
C:=\{\langle t, 0\rangle ; t \in B \& \operatorname{lv}(t) \in \operatorname{Lim}\} .
$$

Then $C$ is a closed subset of $\mathfrak{X}\left(\left\langle T^{n}, \pi^{n} ; n \in \omega\right\rangle\right)$.

For each $\alpha \in \omega_{1} \cap \operatorname{Lim}$, let $t_{\alpha} \in B$ be the node of height $\alpha, s_{\alpha} \in B$ the node of height $\alpha+1$, and let

$$
p_{\alpha}:=\left\langle\left\{\left\langle s_{\alpha}, 0\right\rangle\right\},\left\{\left\langle\left\langle t_{\alpha}, 0\right\rangle, 0\right\rangle\right\}\right\rangle .
$$

Then each $p_{\alpha}$ is a condition of the forcing notion $\mathrm{D}(C)$, and the set $\left\{p_{\alpha} ; \alpha \in\right.$ $\left.\omega_{1} \cap \operatorname{Lim}\right\}$ is an antichain in $\mathrm{D}(C)$, because if $\alpha$ and $\beta$ are countable limit ordinals and $\alpha<\beta$, then $s_{\alpha} \in N\left(\left\langle t_{\beta}, 0\right\rangle, 0\right)$. 
Now we introduce other forcing notions. To do this, we use the following notation and proposition.

Suppose that $\left\langle T^{n} ; n \in \omega\right\rangle$ is a decreasing sequence of $\omega_{1}$-trees. For each $n \in \omega, \mathcal{F}^{n}$ is the set of cofinal chains $\left(^{3}\right)$ in the tree $T^{n} \times\{n\}$,

$$
\mathcal{F}_{+}^{n}:=\left\{B \cap\left(\left(T^{n}\right)_{\geq \alpha} \times\{n\}\right) ; B \in \mathcal{F}^{n} \& \alpha \in \omega_{1}\right\},
$$

and for each $B \in \mathcal{F}_{+}^{n}$,

$$
B^{\downarrow}:=\left\{s \in T^{n} ; \exists\langle t, n\rangle \in B\left(s \leq_{T^{n}} t\right)\right\} \times\{n\} .
$$

Proposition 4.2. Suppose that $\left\langle C_{\nu} ; \nu \in \omega\right\rangle$ is a decreasing sequence of closed subsets with empty intersection. Let $i \in \omega$ and $B \in \mathcal{F}^{i}$. Then there exist $\nu \in \omega$ and $\alpha \in \omega_{1}$ such that $C_{\nu} \cap B \cap\left(T_{\geq \alpha} \times\{i\}\right)=\emptyset$.

Proof. For each $\nu \in \omega$, let

$$
D_{\nu}:=\left\{\operatorname{lv}(s) ;\langle s, i\rangle \in C_{\nu} \cap B\right\} .
$$

Since each $C_{\nu}$ is closed, $D_{\nu}$ is club in $\omega_{1}$ if it is uncountable. So if $D_{\nu}$ is uncountable for every $\nu \in \omega$, then $\bigcap_{\nu \in \omega} D_{\nu}$ is also club in $\omega_{1}$, in particular, it is not empty, hence neither is $\bigcap_{\nu \in \omega} C_{\nu}$.

4.1. Proof of Theorem 4. Throughout this subsection, we suppose that $\left\langle T^{n} ; n \in \omega\right\rangle$ is a decreasing sequence of $\omega_{1}$-trees and $\left\langle\pi^{n} ; n \in \omega \backslash\{0\}\right\rangle$ is a sequence of functions with (p1-3) such that $T^{0}$ has at most countably many cofinal chains.

Let $\left\langle C_{\nu} ; \nu \in \omega\right\rangle$ be a decreasing sequence of closed subsets with empty intersection. Then by Proposition 4.2, there exists $\delta \in \omega_{1}$ such that

- for all $i \in \omega$ and $B, B^{\prime} \in \mathcal{F}^{i}$ with $B \neq B^{\prime}, B \cap B^{\prime} \subseteq\left(T^{i}\right)_{\leq \delta} \times\{i\}$,

- for all $i, \nu \in \omega$ and $B \in \mathcal{F}^{i}$, either $C_{\nu} \cap B$ is uncountable or $C_{\nu} \cap B \cap$ $\left(\left(T^{i}\right)_{\geq \delta} \times\{i\}\right)=\emptyset$.

We introduce the forcing notion

$$
\mathrm{P}\left(\left\langle T^{n}, \pi^{n} ; n \in \omega\right\rangle,\left\langle C_{\nu} ; \nu \in \omega\right\rangle, \delta\right),
$$

or $\mathrm{P}\left(\left\langle C_{\nu} ; \nu \in \omega\right\rangle, \delta\right)$ for short, which is a modification of the forcing notion $\prod_{\nu \in \omega} \mathrm{D}\left(C_{\nu}\right)$ and consists of pairs $\langle\vec{A}, \vec{h}\rangle$ such that:

1. $\vec{A}$ is a finite partial function from $\omega$ into $\left[\bigcup_{n \in \omega} T^{n} \times\{n\}\right]^{<\aleph_{0}}$,

2. $\vec{h}$ is a finite partial function from $\omega \times\left(\bigcup_{n \in \omega} T^{n} \times\{n\}\right)$ into $\omega_{1}$,

3. $\operatorname{dom}(\vec{A}) \supseteq\left\{\nu \in \omega ; \exists x \in \bigcup_{n \in \omega} T^{n} \times\{n\}(\langle\nu, x\rangle \in \operatorname{dom}(\vec{h}))\right\}$,

4. for any $\nu \in \operatorname{dom}(\vec{A})$ and $\langle s, i\rangle \in \vec{A}(\nu)$, if $\operatorname{lv}(s)>\delta$, then either $T^{i}\lceil s$ is Aronszajn or there exists (a unique) $B \in \mathcal{F}^{i}$ with $\langle s, i\rangle \in B$ such that $C_{\nu} \cap B \subseteq\left(T^{i}\right)_{\leq \delta} \times\{i\}$,

$\left({ }^{3}\right)$ In this paper, a cofinal chain means an uncountable subset $B$ of $T$ such that $B$ is pairwise comparable in $T$ and $B \cap T_{\alpha} \neq \emptyset$ for every $\alpha \in \omega_{1}$. 
5. for any $\langle\nu,\langle s, i\rangle\rangle \in \operatorname{dom}(\vec{h})$,

- if $s$ has a successor level, then $\vec{h}(\langle\nu,\langle s, i\rangle\rangle)=\operatorname{lv}(s)-1$,

- if $s$ has a limit level and $\operatorname{lv}(s) \leq \delta$, then $\vec{h}(\langle\nu,\langle s, i\rangle\rangle) \in \operatorname{Iv}(s)$,

- if $s$ has a limit level and $\operatorname{lv}(s)>\delta$, then $\vec{h}(\langle\nu,\langle s, i\rangle\rangle) \in[\delta+1, \operatorname{lv}(s))$,

6. for any $\langle\nu,\langle s, i\rangle\rangle \in \operatorname{dom}(\vec{h})$, either $T^{i}\lceil s$ is Aronszajn or there exists (a unique) $B \in \mathcal{F}^{i}$ with $\langle s, i\rangle \in B$ such that $C_{\nu} \cap B$ is uncountable,

7. for every $\nu \in \operatorname{dom}(\vec{A})$,

$$
\begin{aligned}
(\vec{A}(\nu) \cup \bigcup\{B \cap & \left(\left(T^{i}\right)_{\geq \delta+1} \times\{i\}\right) ; \\
i \in \omega & \left.\left.\& B \in \mathcal{F}^{i} \& C_{\nu} \cap B \text { is countable }\right\}\right) \\
& \cap\left(C_{\nu} \cup \bigcup\{N(x, \vec{h}(\langle\nu, x\rangle)) ;\langle\nu, x\rangle \in \operatorname{dom}(\vec{h})\}\right)=\emptyset,
\end{aligned}
$$

ordered by extensions, that is, for conditions $\langle\vec{A}, \vec{h}\rangle$ and $\left\langle\vec{A}^{\prime}, \vec{h}^{\prime}\right\rangle$,

$$
\langle\vec{A}, \vec{h}\rangle \leq_{\mathrm{P}\left(\left\langle C_{\nu} ; \nu \in \omega\right\rangle, \delta\right)}\left\langle\vec{A}^{\prime}, \vec{h}^{\prime}\right\rangle: \Leftrightarrow \forall \nu \in \operatorname{dom}\left(\vec{A}^{\prime}\right)\left(\vec{A}(\nu) \supseteq \vec{A}^{\prime}(\nu)\right) \& \vec{h} \supseteq \vec{h}^{\prime} .
$$

By a similar $\Delta$-system argument to that in Section 2.1 , we infer that the forcing notion $\mathrm{P}\left(\left\langle T^{n}, \pi^{n} ; n \in \omega\right\rangle,\left\langle C_{\nu} ; \nu \in \omega\right\rangle, \delta\right)$ has the rectangle refining property, and we can prove the following proposition.

Proposition 4.3.

1. For all $v \in \omega_{1}$ and $x \in \bigcup_{n \in \omega} T^{n} \times\{n\}$, the set

$$
\begin{aligned}
\left\{\langle \vec { A } , \vec { h } \rangle \in \mathrm { P } \left(\left\langleC_{\nu} ; \nu\right.\right.\right. & \in \omega\rangle, \delta) ;\langle v, x\rangle \in \operatorname{dom}(\vec{h}) \\
& \text { or } \left.x \notin C_{v} \cup \bigcup\{N(y, \vec{h}(\langle v, y\rangle)) ;\langle v, y\rangle \in \operatorname{dom}(\vec{h})\}\right\}
\end{aligned}
$$

is dense in $\mathrm{P}\left(\left\langle C_{\nu} ; \nu \in \omega\right\rangle, \delta\right)$.

2. For all $x \in \bigcup_{n \in \omega} T^{n} \times\{n\}$, the set

$$
\left\{\langle\vec{A}, \vec{h}\rangle \in \mathrm{P}\left(\left\langle C_{\nu} ; \nu \in \omega\right\rangle, \delta\right) ; \exists \mu \in \operatorname{dom}(\vec{A})(x \in \vec{A}(\mu))\right\}
$$

is dense in $\mathrm{P}\left(\left\langle C_{\nu} ; \nu \in \omega\right\rangle, \delta\right)$.

Suppose that $G$ is a $\mathrm{P}\left(\left\langle C_{\nu} ; \nu \in \omega\right\rangle, \delta\right)$-generic filter, and for each $v \in \omega$, define

$$
\begin{aligned}
& U_{v}:=\bigcup\{N(x, \vec{h}(\langle v, x\rangle)) ; \\
& \left.x \in \bigcup_{n \in \omega} T^{n} \times\{n\} \& \exists\langle\vec{A}, \vec{h}\rangle \in G(\langle v, x\rangle \in \operatorname{dom}(\vec{h}))\right\}, \\
& W_{v}:=\bigcup\{\vec{A}(v) ; \exists\langle\vec{A}, \vec{h}\rangle \in G(v \in \operatorname{dom}(\vec{A}))\} .
\end{aligned}
$$

In view of the above proposition, for each $v \in \omega$, 
- $U_{v}$ is an open set which covers $C_{v}$,

- $W_{v}$ is disjoint from $U_{v}$,

- $\bigcup_{\nu \in \omega} W_{\nu}$ covers the whole space, hence $\bigcap_{\nu \in \omega} U_{\nu}$ is empty.

So Theorem 4 is proved. A similar result also holds for normality, by a similar modification.

4.2. Proof of Theorem 5. Throughout this subsection, we suppose that $\left\langle T^{n} ; n \in \omega\right\rangle$ is a decreasing sequence of $\omega_{1}$-trees and $\left\langle\pi^{n} ; n \in \omega \backslash\{0\}\right\rangle$ is a sequence of functions with (p1-3). We moreover suppose that for all $n \in \omega$,

(u1) the set of cofinal chains in $T^{n}$ is of size $\aleph_{1}$,

(u2) for any countable elementary submodel $M$ of $H\left(\aleph_{2}\right)$ which includes $T^{n}$ and any $t \in\left(T^{n}\right)_{\omega_{1} \cap M}$, there exists a cofinal chain $B$ in $T^{n}$ such that $t \in B \in M$.

Let

$$
\mathbb{Q}_{<\omega_{1}}^{\text {fin }}:=\left\{f \in{ }^{<\omega_{1}} \omega ; \exists k \in \omega(\{\alpha \in \operatorname{dom}(f) ; f(\alpha) \neq k\} \text { is finite })\right\},
$$

which can be considered as a tree ordered by inclusion. $\mathbb{Q}_{<\omega_{1}}^{\text {fin }}$ satisfies (u1-2). In fact, König proved that under PFA, every $\omega_{1}$-tree is either isomorphic to $\mathbb{Q}_{<\omega_{1}}^{\text {fin }}$ or has a stationary antichain [10, part 2.d]. However, there are many counterexamples to (u1-2). We note that if $T^{n}$ satisfies $(\mathbf{u} \mathbf{2})$, then it has uncountably many cofinal chains, and every node of $T^{n}$ has a cofinal chain. But the author is not sure whether (u2) implies (u1). We should note that under PFA, the number of cofinal branches of an $\omega_{1}$-tree is $\leq \aleph_{1}$ (see e.g. $[2, \S 8]$ or $[3, \S 7])$.

Before defining the forcing notion in this subsection, we record some relationships between $\omega_{1}$-trees and countable elementary submodels of $H\left(\aleph_{2}\right)$.

Observation 4.4. Suppose that $T$ is an $\omega_{1}$-tree, $M$ is a countable elementary submodel of $H\left(\aleph_{2}\right)$ with $T \in \omega_{1}$, and $B$ and $B^{\prime}$ are different cofinal chains in $T$ such that $B$ and $B^{\prime}$ are both members of $M$. Let $b$ and $b^{\prime}$ be nodes in $B$ and $B^{\prime}$ respectively of height $\omega_{1} \cap M$. Then $b$ and $b^{\prime}$ are also different.

This says that if $T$ is an $\omega_{1}$-tree with the property $(\mathbf{u} \mathbf{2})$, and $M$ is a countable elementary submodel with $T \in M$, then for each $t \in T_{\omega_{1} \cap M}$, a cofinal chain through $t$ which belongs to $M$ is unique.

ObSERVATION 4.5. Suppose that $T$ is an $\omega_{1}$-tree with the property (u2), $C$ is a closed subset of $T, M$ is a countable elementary submodel of $H\left(\aleph_{2}\right)$ with $T, C \in \omega_{1} \cap M$, and $B$ is a cofinal chain in $T$ which is a member of $M$. Then there exists $t \in B \cap C$ of height $\geq \omega_{1} \cap M$ iff the set $\{\operatorname{lv}(t) ; t \in B \cap C\}$ is club in $\omega_{1}$ iff $B \cap C \cap T_{\omega_{1} \cap M} \neq \emptyset$. 
Proof. As in the proof of Proposition 4.2, the set $\{\operatorname{lv}(t) ; t \in B \cap C\}$ is club in $\omega_{1}$ if it is uncountable. So if it is not, since $\{\operatorname{lv}(t) ; t \in B \cap C\}$ is also a member of $M$, there exists $\alpha \in \omega_{1} \cap M$ such that $\{\operatorname{lv}(t) ; t \in B \cap C\} \subseteq \alpha$, so there are no $t \in B \cap C$ of height $\geq \omega_{1} \cap M$.

If $\{\operatorname{lv}(t) ; t \in B \cap C\}$ is club in $\omega_{1}$, then $\left\{\operatorname{lv}(t) \in \omega_{1} \cap M ; t \in B \cap C \cap M\right\}$ is unbounded in $\omega_{1} \cap M$. Thus then $B \cap C \cap T_{\omega_{1} \cap M} \neq \emptyset$.

Let $\left\langle C_{\nu} ; \nu \in \omega\right\rangle$ be a decreasing sequence of closed subsets with empty intersection. We introduce a forcing notion

$$
\mathrm{Q}\left(\left\langle T^{n}, \pi^{n} ; n \in \omega\right\rangle,\left\langle C_{\nu} ; \nu \in \omega\right\rangle\right),
$$

or $\mathrm{Q}\left(\left\langle C_{\nu} ; \nu \in \omega\right\rangle\right)$ for short, which can be considered as one of applications of Todorčević's side condition method [22, §8] and consists of tuples $\langle\vec{A}, \overrightarrow{\mathcal{B}}, \vec{h}, \overrightarrow{\mathcal{C}}, \mathcal{N}\rangle$ such that:

1. $\vec{A}$ is a finite partial function from $\omega$ into $\left[\bigcup_{n \in \omega} T^{n} \times\{n\}\right]^{<\aleph_{0}}$,

2. $\overrightarrow{\mathcal{B}}$ and $\overrightarrow{\mathcal{C}}$ are finite partial functions from $\omega$ into $\left[\bigcup_{n \in \omega} \mathcal{F}_{+}^{n}\right]<\aleph_{0}$,

3. $\vec{h}$ is a finite partial function from $\omega \times\left(\bigcup_{n \in \omega} T^{n} \times\{n\}\right)$ into $\omega_{1}$,

4. $\mathcal{N}$ is a finite $\in$-chain of countable elementary submodels of $H\left(\aleph_{2}\right)$ containing $\left\langle T^{n}, \pi^{n}, \mathcal{F}^{n} ; n \in \omega\right\rangle,\left\langle C_{\nu} ; \nu \in \omega\right\rangle$ and $\omega_{1}$,

5. $\operatorname{dom}(\vec{A})=\operatorname{dom}(\overrightarrow{\mathcal{B}})=\operatorname{dom}(\overrightarrow{\mathcal{C}})$

$$
\supseteq\left\{\nu \in \omega ; \exists x \in \bigcup_{n \in \omega} T^{n} \times\{n\}(\langle\nu, x\rangle \in \operatorname{dom}(\vec{h}))\right\},
$$

6. for any $\nu \in \operatorname{dom}(\vec{A}),\langle s, i\rangle \in \vec{A}(\nu)$ and $N \in \mathcal{N}$, if $s \notin N$, then for all $B \in \mathcal{F}^{i} \cap N,\langle s, i\rangle \notin B$,

7. for any $\nu \in \operatorname{dom}(\vec{A}), B \in \overrightarrow{\mathcal{B}}(\nu)$ and $N \in \mathcal{N}$, if $B \notin N$, then there exists $B^{\prime} \in \mathcal{B}(\nu) \cap N$ such that $B^{\downarrow} \cap N=\left(B^{\prime}\right)^{\downarrow} \cap N$ and $B \cap N \subseteq$ $B^{\prime} \cap N$,

8. for any $\nu \in \operatorname{dom}(\vec{A}), B \in \overrightarrow{\mathcal{C}}(\nu)$ and $N \in \mathcal{N}$, if $B^{\downarrow} \notin N$ and $B \cap N \neq \emptyset$, then there exists $B^{\prime} \in \overrightarrow{\mathcal{C}}(\nu) \cap N$ such that $B \cap N=B^{\prime} \cap N$,

9. for any $\langle\nu,\langle s, i\rangle\rangle \in \operatorname{dom}(\vec{h})$, if $s$ has a successor level, then $\vec{h}(\langle\nu,\langle s, i\rangle\rangle)$ $=\operatorname{lv}(s)-1$, otherwise $\vec{h}(\langle\nu,\langle s, i\rangle\rangle) \in \operatorname{lv}(s)$,

10. for each $\langle\nu,\langle s, i\rangle\rangle \in \operatorname{dom}(\vec{h})$, if there exists $N \in \mathcal{N}$ with $s \notin N$ and $\vec{h}(\langle\nu,\langle s, i\rangle\rangle) \in N$, then there exists $B \in \overrightarrow{\mathcal{C}}(\nu) \cap N$ such that

$$
N(\langle s, i\rangle, \vec{h}(\langle\nu,\langle s, i\rangle\rangle)) \cap\left(T^{i} \times\{i\}\right) \subseteq B,
$$

11. for any $\nu \in \operatorname{dom}(\vec{A})$,

$$
\begin{aligned}
(\vec{A}(\nu) & \cup \bigcup \overrightarrow{\mathcal{B}}(\nu)) \\
& \cap\left(C_{\nu} \cup \bigcup\{N(x, \vec{h}(\langle\nu, x\rangle)) ;\langle\nu, x\rangle \in \operatorname{dom}(\vec{h})\} \cup \bigcup \overrightarrow{\mathcal{C}}(\nu)\right)=\emptyset,
\end{aligned}
$$


ordered by extensions, that is, for conditions $\langle\vec{A}, \overrightarrow{\mathcal{B}}, \vec{h}, \overrightarrow{\mathcal{C}}, \mathcal{N}\rangle$ and $\left\langle\overrightarrow{A^{\prime}}, \overrightarrow{\mathcal{B}^{\prime}}, \vec{h}^{\prime}\right.$, $\left.\overrightarrow{\mathcal{C}^{\prime}}, \mathcal{N}^{\prime}\right\rangle$,

$$
\begin{aligned}
\langle\vec{A}, \overrightarrow{\mathcal{B}}, \vec{h}, \overrightarrow{\mathcal{C}}, \mathcal{N}\rangle \leq_{\mathrm{Q}}\left(\left\langle C_{\nu} ; \nu \in \omega\right\rangle\right) & \left\langle\vec{A}^{\prime}, \overrightarrow{\mathcal{B}}^{\prime}, \vec{h}^{\prime}, \overrightarrow{\mathcal{C}^{\prime}}, \mathcal{N}^{\prime}\right\rangle \\
: \Leftrightarrow & \operatorname{dom}(\vec{A}) \supseteq \operatorname{dom}\left(\vec{A}^{\prime}\right) \text { and for all } \nu \in \operatorname{dom}\left(\overrightarrow{A^{\prime}}\right), \\
& \vec{A}(\nu) \supseteq \vec{A}^{\prime}(\nu), \overrightarrow{\mathcal{B}}(\nu) \supseteq \overrightarrow{\mathcal{B}}^{\prime}(\nu) \text { and } \overrightarrow{\mathcal{C}}(\nu) \supseteq \overrightarrow{\mathcal{C}}^{\prime}(\nu), \\
& \vec{h} \supseteq \vec{h}^{\prime} \text { and } \mathcal{N} \supseteq \mathcal{N}^{\prime} .
\end{aligned}
$$

It follows from the above observations that this definition should work well. In fact, the following proposition guarantees that.

Proposition 4.6. For all $v \in \omega$ and $x \in \bigcup_{n \in \omega} T^{n} \times\{n\}$, the set

$$
\left\{\langle\vec{A}, \overrightarrow{\mathcal{B}}, \vec{h}, \overrightarrow{\mathcal{C}}, \mathcal{N}\rangle \in \mathrm{Q}\left(\left\langle C_{\nu} ; \nu \in \omega\right\rangle\right) ;\langle v, x\rangle \in \operatorname{dom}(\vec{h})\right.
$$

$$
\text { or } \left.x \notin C_{v} \cup \bigcup\{N(x, \vec{h}(\langle v, x\rangle)) ;\langle v, x\rangle \in \operatorname{dom}(\vec{h})\} \cup \bigcup \overrightarrow{\mathcal{C}}(v)\right\}
$$

is dense in $\mathrm{Q}\left(\left\langle C_{\nu} ; \nu \in \omega\right\rangle\right)$.

Proof. Let $p=\langle\vec{A}, \overrightarrow{\mathcal{B}}, \vec{h}, \overrightarrow{\mathcal{C}}, \mathcal{N}\rangle, v \in \omega$ and $x=\langle s, i\rangle$, and assume that

$$
x \in C_{v} \cup \bigcup\{N(x, \vec{h}(\langle v, x\rangle)) ;\langle v, x\rangle \in \operatorname{dom}(\vec{h})\} \cup \bigcup \overrightarrow{\mathcal{C}}(v) .
$$

Since $x \notin \vec{A}(v) \cup \bigcup \overrightarrow{\mathcal{B}}(v)$ and both $\vec{A}(v)$ and $\overrightarrow{\mathcal{B}}(v)$ are finite, there exists $\alpha \in \operatorname{lv}(s)$ such that

$$
(\vec{A}(v) \cup \bigcup \overrightarrow{\mathcal{B}}(v)) \cap N(x, \alpha)=\emptyset,
$$

and for any $N \in \mathcal{N}$ with $\operatorname{lv}(s)>\omega_{1} \cap N$, the inequality $\alpha>\omega_{1} \cap N$ holds. If there are no $N \in \mathcal{N}$ with $\operatorname{lv}(s)=\omega_{1} \cap N$, then we need nothing more to make the desired extension.

Suppose that there is $N \in \mathcal{N}$ with $\operatorname{lv}(s)=\omega_{1} \cap N$. Then by Observation 4.5 , there are no $B \in \overrightarrow{\mathcal{B}}(v)$ with $x \in B^{\downarrow}$. Then there exists (a unique) $B_{0} \in \mathcal{F}^{i} \cap N$ with $x \in B_{0}$. Notice that $\left\{\operatorname{lv}(u) ;\langle u, i\rangle \in B_{0} \cap C_{v}\right\}$ is club in $\omega_{1}$, so we cannot put any subset of $B_{0}$ into the coordinate $\overrightarrow{\mathcal{B}}(v)$. Now we assume that $\nu \in \operatorname{dom}(\vec{A})$. (If not, we let $\vec{A}(\nu)=\overrightarrow{\mathcal{B}}(\nu)=\emptyset$.) Define

$$
B_{0}^{\prime}:=B_{0} \cap\left(\left(T^{i}\right)_{\geq \alpha+1} \times\{i\}\right) .
$$

Then the tuple

$$
\left\langle\vec{A}, \overrightarrow{\mathcal{B}}, \vec{h} \cup\{\langle\langle v, x\rangle, \alpha\rangle\},(\overrightarrow{\mathcal{C}} \backslash\{\langle v, \overrightarrow{\mathcal{C}}(v)\rangle\}) \cup\left\{\left\langle v, \overrightarrow{\mathcal{C}}(v) \cup\left\{B_{0}^{\prime}\right\}\right\rangle\right\}, \mathcal{N}\right\rangle
$$

is a condition of $\mathrm{Q}\left(\left\langle C_{\nu} ; \nu \in \omega\right\rangle\right)$ and an extension of $p$.

Proposition 4.7. For all $x \in \bigcup_{n \in \omega} T^{n} \times\{n\}$, the set

$$
\left\{\langle\vec{A}, \overrightarrow{\mathcal{B}}, \vec{h}, \overrightarrow{\mathcal{C}}, \mathcal{N}\rangle \in \mathrm{Q}\left(\left\langle C_{\nu} ; \nu \in \omega\right\rangle\right) ; \exists \mu \in \operatorname{dom}(\vec{A})(x \in \vec{A}(\mu) \cup \bigcup \overrightarrow{\mathcal{B}}(\mu))\right\}
$$

is dense in $\mathrm{Q}\left(\left\langle C_{\nu} ; \nu \in \omega\right\rangle\right)$. 
Proof. Let $p=\langle\vec{A}, \overrightarrow{\mathcal{B}}, \vec{h}, \overrightarrow{\mathcal{C}}, \mathcal{N}\rangle$ and $x=\langle s, i\rangle$. Since $\operatorname{dom}(\vec{A})$ is finite, there exists $\nu \in \omega$ such that $\operatorname{dom}(\vec{A}) \subseteq \nu$.

Suppose that $x \notin B$ for any $N \in \mathcal{N}$ and $B \in \mathcal{F}^{i} \cap N$. Let $\mu \geq \nu$ be such that $x \notin C_{\mu}$. Then the tuple

$$
\langle\vec{A} \cup\{\langle\mu,\{x\}\rangle\}, \overrightarrow{\mathcal{B}} \cup\{\langle\mu,\{\emptyset\}\rangle\}, \vec{h}, \overrightarrow{\mathcal{C}} \cup\{\langle\mu,\{\emptyset\}\rangle\}, \mathcal{N}\rangle
$$

is a condition of $\mathrm{Q}\left(\left\langle C_{\nu} ; \nu \in \omega\right\rangle\right)$ and an extension of $p$.

Suppose that there exist $N \in \mathcal{N}$ and $B \in \mathcal{F}^{i} \cap N$ such that $x \in B \backslash N$. Let $\left\langle N_{j} ; j \in k\right\rangle$ be an enumeration of $\mathcal{N}$ such that $N_{j} \in N_{j+1}$ for each $j \in k-1$, and let $l \in k$ be the largest number such that $x \notin N_{l}$. Then we can find a finite subset $\left\{B_{j} ; j \in l+1\right\}$ of $\mathcal{F}^{i}$ such that

- for each $j \in l+1, B_{j} \in N_{j}$,

- for any $j, j^{\prime} \in l+1$ with $j<j^{\prime}, B_{j} \cap N_{j}=B_{j^{\prime}} \cap N_{j}$,

- $x \in B_{l}$.

Then we can find $\mu \geq \nu$ and an increasing sequence $\left\langle\alpha_{j} ; j \in l+1\right\rangle$ such that for each $j \in l+1$ we have $\alpha_{j} \in \omega_{1} \cap N_{j}$ and

$$
C_{\mu} \cap B_{j} \cap\left(\left(T^{i}\right)_{\geq \alpha_{j}} \times\{i\}\right)=\emptyset .
$$

Then the tuple

$$
\begin{array}{r}
\left\langle\vec{A} \cup\{\langle\mu,\{\emptyset\}\rangle\}, \overrightarrow{\mathcal{B}} \cup\left\{\left\langle\mu, \overrightarrow{\mathcal{B}}(\mu) \cup\left\{B_{j} \cap\left(\left(T^{i}\right)_{\geq \alpha_{j}} \times\{i\}\right) ; j \in l+1\right\}\right\rangle\right\},\right. \\
\vec{h}, \overrightarrow{\mathcal{C}} \cup\{\langle\mu,\{\emptyset\}\rangle\}, \mathcal{N}\rangle
\end{array}
$$

is a condition of $\mathrm{Q}\left(\left\langle C_{\nu} ; \nu \in \omega\right\rangle\right)$ and an extension of $p$.

Suppose that $G$ is a $\mathrm{Q}\left(\left\langle C_{\nu} ; \nu \in \omega\right\rangle\right)$-generic filter, and for each $v \in \omega$, define

$$
\begin{aligned}
& U_{v}:=\bigcup\{N(x, \vec{h}(\langle v, x\rangle)) ; \\
& \left.\quad x \in \bigcup_{n \in \omega} T^{n} \times\{n\} \& \exists\langle\vec{A}, \overrightarrow{\mathcal{B}}, \vec{h}, \overrightarrow{\mathcal{C}}, \mathcal{N}\rangle \in G(\langle v, x\rangle \in \operatorname{dom}(\vec{h}))\right\}, \\
& W_{v}:=\bigcup\{\vec{A}(v) ; \exists\langle\vec{A}, \overrightarrow{\mathcal{B}}, \vec{h}, \overrightarrow{\mathcal{C}}, \mathcal{N}\rangle \in G(v \in \operatorname{dom}(\vec{A}))\} .
\end{aligned}
$$

In view of the above two propositions, for each $v \in \omega$,

- $U_{v}$ is an open set which covers $C_{v}$,

- $W_{v}$ is disjoint from $U_{v}$,

- $\bigcup_{\nu \in \omega} W_{\nu}$ covers the whole space, hence $\bigcap_{\nu \in \omega} U_{\nu}$ is empty.

Lemma 4.8. $\mathrm{Q}\left(\left\langle T^{n}, \pi^{n} ; n \in \omega\right\rangle,\left\langle C_{\nu} ; \nu \in \omega\right\rangle\right)$ is proper.

Proof. Let $M$ be a countable elementary submodel of $H(\kappa)$, where $\kappa$ is a large enough regular cardinal, such that $\left\langle T^{n}, \pi^{n} ; n \in \omega\right\rangle,\left\langle C_{\nu} ; \nu \in \omega\right\rangle$, $\omega_{1}, H\left(\aleph_{2}\right)$ and a well-ordering of $H\left(\aleph_{2}\right)$ are elements of $M$, and let $p=$ $\left\langle\overrightarrow{A_{p}}, \overrightarrow{\mathcal{B}_{p}}, \vec{h}_{p}, \overrightarrow{\mathcal{C}}_{p}, \mathcal{N}_{p}\right\rangle$ be a condition of $\mathrm{Q}\left(\left\langle C_{\nu} ; \nu \in \omega\right\rangle\right) \cap M$. Then by taking 
the Skolem hull of the set

$$
\left\{p,\left\langle T^{n}, \pi^{n} ; n \in \omega\right\rangle,\left\langle C_{\nu} ; \nu \in \omega\right\rangle, \omega_{1}\right\}
$$

on $H\left(\aleph_{2}\right)$ in $M$, we can find a countable elementary submodel $M_{0}$ of $H\left(\aleph_{2}\right)$ in $M$ containing $p,\left\langle T^{n}, \pi^{n} ; n \in \omega\right\rangle,\left\langle C_{\nu} ; \nu \in \omega\right\rangle$ and $\omega_{1}$. Note that $M \cap H\left(\aleph_{2}\right)$ is an elementary submodel of $H\left(\aleph_{2}\right)$. Let

$$
p^{+}:=\left\langle\vec{A}_{p}, \overrightarrow{\mathcal{B}}_{p}, \vec{h}_{p}, \overrightarrow{\mathcal{C}}_{p}, \mathcal{N}_{p} \cup\left\{M_{0}, M \cap H\left(\aleph_{2}\right)\right\}\right\rangle .
$$

We show that $p^{+}$is $\left(M, \mathrm{Q}\left(\left\langle C_{\nu} ; \nu \in \omega\right\rangle\right)\right)$-generic.

Suppose that $\mathcal{D} \in M$ is a dense subset of $\mathrm{Q}\left(\left\langle C_{\nu} ; \nu \in \omega\right\rangle\right)$ in $M$, and $q$ is a condition of $\mathrm{Q}\left(\left\langle C_{\nu} ; \nu \in \omega\right\rangle\right)$ such that

$$
q=\left\langle\overrightarrow{A_{q}}, \overrightarrow{\mathcal{B}_{q}}, \vec{h}_{q}, \overrightarrow{\mathcal{C}}_{q}, \mathcal{N}_{q}\right\rangle \leq_{\mathrm{Q}\left(\left\langle C_{\nu} ; \nu \in \omega\right\rangle\right)} p^{+} .
$$

By strengthening $q$ if necessary, we may assume that

- $q \in \mathcal{D}$,

- for every $\langle\nu,\langle s, i\rangle\rangle \in \operatorname{dom}\left(\vec{h}_{q}\right) \backslash M$, either $\left\langle s \mid \vec{h}_{q}(\langle\nu,\langle s, i\rangle\rangle)+1, i\right\rangle \in B$ for some $B \in \overrightarrow{\mathcal{C}}_{q}(\nu)$, or $\left\langle s \mid \vec{h}_{q}(\langle\nu,\langle s, i\rangle\rangle)+1, i\right\rangle \notin B$ for any $B \in \mathcal{F}^{i} \cap M$.

(Indeed, if there exists $B \in \mathcal{F}^{i} \cap M$ such that $\left\langle s \mid \vec{h}_{q}(\langle\nu,\langle s, i\rangle\rangle)+1, i\right\rangle \in B$, then $\vec{A}_{q}(\nu) \cap B=\emptyset$ and for any $\alpha \in \omega_{1}, B \cap\left(T^{i} \geq \alpha \times\{i\}\right) \notin \overrightarrow{\mathcal{B}}_{q}(\nu)$ by condition 7 in the definition of $\mathrm{Q}\left(\left\langle C_{\nu} ; \nu \in \omega\right\rangle\right)$. Note that if $B \in \mathcal{F}_{+}^{i} \cap M$, then $B=B^{\downarrow} \cap\left(\left(T^{i}\right)_{>\alpha} \times\{i\}\right)$ for some $\alpha \in \omega_{1} \cap M$.)

Let $q^{\prime}:=\left\langle\vec{A}_{q^{\prime}}, \overrightarrow{\mathcal{B}}_{q^{\prime}}, \vec{h}_{q^{\prime}}, \overrightarrow{\mathcal{C}}_{q^{\prime}}, \mathcal{N}_{q^{\prime}}\right\rangle$ be such that:

- $\operatorname{dom}\left(\vec{A}_{q^{\prime}}\right):=\operatorname{dom}\left(\vec{A}_{q}\right)$ and $\vec{A}_{q^{\prime}}(\nu):=\vec{A}_{q}(\nu) \cap M$ for each $\nu \in \operatorname{dom}\left(\vec{A}_{q^{\prime}}\right)$,

- $\operatorname{dom}\left(\overrightarrow{\mathcal{B}}_{q^{\prime}}\right):=\operatorname{dom}\left(\overrightarrow{\mathcal{B}_{q}}\right)$ and $\overrightarrow{\mathcal{B}}_{q^{\prime}}(\nu):=\overrightarrow{\mathcal{B}}_{q}(\nu) \cap M$ for each $\nu \in \operatorname{dom}\left(\overrightarrow{\mathcal{B}}_{q^{\prime}}\right)$,

- $\vec{h}_{q^{\prime}}:=\vec{h}_{q} \cap M$,

- $\operatorname{dom}\left(\overrightarrow{\overrightarrow{\mathcal{C}}_{q^{\prime}}}\right):=\operatorname{dom}\left(\overrightarrow{\mathcal{C}_{q}}\right)$ and $\overrightarrow{\mathcal{C}}_{q^{\prime}}(\nu):=\overrightarrow{\mathcal{C}}_{q}(\nu) \cap M$ for each $\nu \in \operatorname{dom}\left(\overrightarrow{\overrightarrow{\mathcal{C}}_{q^{\prime}}}\right)$,

- $\mathcal{N}_{q^{\prime}}:=\mathcal{N}_{q} \cap M$.

We note that $q^{\prime} \in M$ and $M_{0} \in \mathcal{N}_{q^{\prime}}$, in particular, $\mathcal{N}_{q^{\prime}}$ is not empty, hence for each $\nu \in \operatorname{dom}\left(\vec{A}_{q}\right), \overrightarrow{\mathcal{B}}_{q^{\prime}}(\nu)$ is empty iff $\overrightarrow{\mathcal{B}}_{q}(\nu)$ is. Let $M_{1} \in M$ be the Skolem hull of the set

$$
\left\{q^{\prime},\left\langle T^{n}, \pi^{n} ; n \in \omega\right\rangle,\left\langle C_{\nu} ; \nu \in \omega\right\rangle, \omega_{1}\right\}
$$

on $H\left(\aleph_{2}\right)$ in $M$. Now we let

$$
\begin{aligned}
\sigma & :=\left\{\langle\nu, i\rangle \in \operatorname{dom}\left(\vec{A}_{q}\right) \times \omega ; \emptyset \neq \overrightarrow{\mathcal{B}}_{q}(\nu) \cap \mathcal{F}_{+}^{i} \subseteq M\right\}, \\
\tau & :=\left\{\langle\nu, i\rangle \in \operatorname{dom}\left(\vec{A}_{q}\right) \times \omega ;\left(\overrightarrow{\mathcal{B}}_{q}(\nu) \cap \mathcal{F}_{+}^{i}\right) \backslash M \neq \emptyset\right\} .
\end{aligned}
$$

Then both $\sigma$ and $\tau$ are finite and so members of $M$, and for each $\langle\nu, i\rangle \in \sigma$,

$$
\overrightarrow{\mathcal{B}}_{q^{\prime}}(\nu) \cap \mathcal{F}_{+}^{i}=\overrightarrow{\mathcal{B}}_{q}(\nu) \cap \mathcal{F}_{+}^{i} .
$$


For each $\langle\nu, i\rangle \in \tau$ and $B \in\left(\overrightarrow{\mathcal{B}}_{q}(\nu) \cap \mathcal{F}_{+}^{i}\right) \backslash M$, there exists $B^{\prime} \in \overrightarrow{\mathcal{B}}_{q^{\prime}}(\nu)$ such that $B^{\downarrow} \cap M=\left(B^{\prime}\right)^{\downarrow} \cap M$ and $B \cap M \subseteq B^{\prime} \cap M$. Thus $B^{\prime} \cap M$ is not empty and

$$
B^{\prime} \cap\left(\bigcup_{x \in \operatorname{dom}\left(\vec{h}_{q}\right)(\nu)} N\left(x, \vec{h}_{q}(\langle\nu, x\rangle)\right) \cup \bigcup \overrightarrow{\mathcal{C}}_{q}(\nu)\right)=\emptyset .
$$

We will find a condition in $\mathcal{D} \cap M$ which is compatible with $q$. First, we will establish when a condition $r=\left\langle\vec{A}_{r}, \overrightarrow{\mathcal{B}}_{r}, \vec{h}_{r}, \overrightarrow{\mathcal{C}}_{r}, \mathcal{N}_{r}\right\rangle$ in $\mathrm{Q}\left(\left\langle C_{\nu} ; \nu \in \omega\right\rangle\right) \cap M$ with the properties

(i) $r \leq_{\mathrm{Q}\left(\left\langle C_{\nu} ; \nu \in \omega\right\rangle\right)} q^{\prime}$,

(ii) for all $\nu \in \operatorname{dom}\left(\vec{A}_{q^{\prime}}\right)$ and $B \in \overrightarrow{\mathcal{B}}_{r}(\nu)$, there exists $\langle\nu, i\rangle \in \sigma \cup \tau$ such that $B \in \mathcal{F}^{i}$,

(iii) for all $\langle\nu, i\rangle \in \sigma, \overrightarrow{\mathcal{B}}_{r}(\nu)=\overrightarrow{\mathcal{B}}_{q^{\prime}}(\nu)$,

(iv) for all $\langle\nu, i\rangle \in \tau, \overrightarrow{\mathcal{B}}_{r}(\nu) \cap M_{1}=\overrightarrow{\mathcal{B}}_{q^{\prime}}(\nu)$,

(v) $\mathcal{N}_{r} \cap M_{1}=\mathcal{N}_{q^{\prime}}$,

is compatible with $q$. Let $r$ be a condition as above. Then for each $\nu \in$ $\operatorname{dom}\left(\vec{A}_{q^{\prime}}\right)$, since

$$
\begin{array}{r}
\left(\bigcup\left\{N\left(x, \vec{h}_{q}(\langle\nu, x\rangle)\right) ;\langle\nu, x\rangle \in \operatorname{dom}\left(\vec{h}_{q}\right) \backslash M\right\} \cup \bigcup\left(\overrightarrow{\mathcal{C}}_{q}(\nu) \backslash M\right)\right) \cap M \\
\subseteq \bigcup\left\{N\left(x, \vec{h}_{q^{\prime}}(\langle\nu, x\rangle)\right) ;\langle\nu, x\rangle \in \operatorname{dom}\left(\vec{h}_{q^{\prime}}\right)\right\} \cup \bigcup \overrightarrow{\mathcal{C}}_{q^{\prime}}(\nu)
\end{array}
$$

and

$$
\bigcup\left\{N\left(x, \vec{h}_{r}(\langle\nu, x\rangle)\right) ;\langle\nu, x\rangle \in \operatorname{dom}\left(\vec{h}_{r}\right)\right\} \subseteq M,
$$

we have

$$
\vec{A}_{r}(\nu) \cap\left(\bigcup\left\{N\left(x, \vec{h}_{q}(\langle\nu, x\rangle)\right) ;\langle\nu, x\rangle \in \operatorname{dom}\left(\vec{h}_{q}\right)\right\} \cup \bigcup \overrightarrow{\mathcal{C}}_{q}(\nu)\right)=\emptyset
$$

and

$$
\vec{A}_{q}(\nu) \cap \bigcup\left\{N\left(x, \vec{h}_{r}(\langle\nu, x\rangle)\right) ;\langle\nu, x\rangle \in \operatorname{dom}\left(\vec{h}_{r}\right)\right\}=\emptyset .
$$

Moreover, for each $\nu \in \operatorname{dom}\left(\vec{A}_{q^{\prime}}\right)$, since $\bigcup \overrightarrow{\mathcal{B}}_{q}(\nu) \cap M=\bigcup \overrightarrow{\mathcal{B}}_{q^{\prime}}(\nu) \cap M$, we have

$$
\bigcup \overrightarrow{\mathcal{B}}_{q}(\nu) \cap \bigcup\left\{N\left(x, \vec{h}_{r}(\langle\nu, x\rangle)\right) ;\langle\nu, x\rangle \in \operatorname{dom}\left(\vec{h}_{r}\right)\right\}=\emptyset,
$$

and by Observations 4.4 and 4.5 and the definition of $\mathrm{Q}\left(\left\langle C_{\nu} ; \nu \in \omega\right\rangle\right)$,

$$
\left(\overrightarrow{A_{q}}(\nu) \cup \bigcup \overrightarrow{\mathcal{B}_{q}}(\nu)\right) \cap \bigcup \overrightarrow{\mathcal{C}}_{r}(\nu)=\emptyset, \quad \bigcup \overrightarrow{\mathcal{B}}_{r}(\nu) \cap \bigcup \overrightarrow{\mathcal{C}_{q}}(\nu)=\emptyset .
$$

Therefore for a condition $r \in \mathrm{Q}\left(\left\langle C_{\nu} ; \nu \in \omega\right\rangle\right) \cap M$ with the properties (i) $-(\mathbf{v})$, if

$$
\bigcup \overrightarrow{\mathcal{B}}_{r}(\nu) \cap \bigcup\left\{N\left(x, \vec{h}_{q}(\langle\nu, x\rangle)\right) ;\langle\nu, x\rangle \in \operatorname{dom}\left(\vec{h}_{q}\right)\right\}=\emptyset,
$$

then $r$ and $q$ are compatible in $\mathrm{Q}\left(\left\langle C_{\nu} ; \nu \in \omega\right\rangle\right)$. 
Now suppose that there exist $\langle\nu,\langle s, j\rangle\rangle \in \operatorname{dom}\left(\vec{h}_{q}\right) \backslash M_{1}$ and $B \in\left(\overrightarrow{\mathcal{B}}_{r}(\nu)\right.$ $\left.\cap \mathcal{F}^{i}\right) \backslash \overrightarrow{\mathcal{B}}_{q^{\prime}}(\nu)$ for some $i \in \omega$ such that

$$
B \cap N\left(\langle s, j\rangle, \vec{h}_{q}(\langle\nu,\langle s, j\rangle\rangle)\right) \neq \emptyset .
$$

Then $\vec{h}_{q}(\langle\nu,\langle s, j\rangle\rangle) \geq \omega_{1} \cap M$ and $B \in M \backslash M_{1}$, so there exists $B^{\prime} \in \overrightarrow{\mathcal{B}}_{q^{\prime}}(\nu)$ such that $B^{\downarrow} \cap M_{1}=\left(B^{\prime}\right)^{\downarrow} \cap M_{1}$ and $B \cap M_{1} \subseteq B^{\prime} \cap M_{1}$, and so $B^{\downarrow} \neq\left(B^{\prime}\right)^{\downarrow}$ and $\langle\nu, i\rangle \in \tau$. Thus now $i+1=j$. (If neither $i=j$ nor $i+1=j$, then by the definition of $N(z, \beta), B \cap N\left(\langle s, j\rangle, \vec{h}_{q}(\langle\nu,\langle s, j\rangle\rangle)\right)=\emptyset$. If $i=j$ and $B \cap N\left(\langle s, j\rangle, \vec{h}_{q}(\langle\nu,\langle s, j\rangle\rangle)\right) \neq \emptyset$, then $\left\langle s \uparrow\left(\vec{h}_{q}(\langle\nu,\langle s, j\rangle\rangle)+1\right), j\right\rangle$ belongs to $B$. This contradicts our assumption on $q$ because $B \in M$.) So then $\Delta(s, B)$ is in the set

$$
\left\{\Delta(u, s) ; u \in \pi^{j}(s)\right\} \backslash \vec{h}_{q}(\langle\nu,\langle s, j\rangle\rangle) .
$$

(Here, we use notation like $\Delta(s, B)$ and $\Delta\left(B, B^{\prime}\right)$, defined in a similar fashion.) Since $\Delta\left(B, B^{\prime}\right) \in \omega_{1} \cap M$, if $\Delta\left(s, B^{\prime}\right) \geq \omega_{1} \cap M$ then $\Delta(s, B)=$ $\Delta\left(B, B^{\prime}\right)<\omega_{1} \cap M$, hence $B \cap N\left(\langle s, j\rangle, \vec{h}_{q}(\langle\nu,\langle s, j\rangle\rangle)\right)=\emptyset$. Even if $\Delta\left(s, B^{\prime}\right)$ $<\omega_{1} \cap M$, whenever $\Delta\left(s, B^{\prime}\right)<\Delta\left(B, B^{\prime}\right)$ then $\Delta(s, B)=\Delta\left(s, B^{\prime}\right)<\omega_{1} \cap M$, hence also $B \cap N\left(\langle s, j\rangle, \vec{h}_{q}(\langle\nu,\langle s, j\rangle\rangle)\right)=\emptyset$. So it suffices to find $r \in \mathcal{D} \cap M$ with properties $(\mathbf{i})-(\mathbf{v})$ and

(vi) for all $\langle\nu, i\rangle \in \tau,\langle\nu,\langle s, i+1\rangle\rangle \in \operatorname{dom}\left(\vec{h}_{q}\right) \backslash M_{1}, B \in\left(\overrightarrow{\mathcal{B}}_{r}(\nu) \cap\right.$ $\left.\mathcal{F}_{+}^{i}\right) \backslash M_{1}$ and $B^{\prime} \in \overrightarrow{\mathcal{B}}_{q^{\prime}}(\nu) \cap \mathcal{F}_{+}^{i}$ with $B^{\downarrow} \cap M_{1}=\left(B^{\prime}\right)^{\downarrow} \cap M_{1}$, if $\Delta\left(s, B^{\prime}\right)<\omega_{1} \cap M$, then either $B \subseteq B^{\prime}$ or $\Delta\left(s, B^{\prime}\right)<\Delta\left(B, B^{\prime}\right)$.

We take $\delta \in \omega_{1} \cap M$ such that for all $\langle\nu, i\rangle \in \tau$ with $\langle\nu,\langle s, i+1\rangle\rangle \in$ $\operatorname{dom}\left(\vec{h}_{q}\right) \backslash M_{1}$, and $B^{\prime} \in \overrightarrow{\mathcal{B}}_{q^{\prime}}(\nu) \cap \mathcal{F}_{+}^{i}$, if $\Delta\left(s, B^{\prime}\right)<\omega_{1} \cap M$ then $\Delta\left(s, B^{\prime}\right)<\delta$. Let

$\mathcal{D}^{-}:=\{\langle\vec{A}, \overrightarrow{\mathcal{B}}, \vec{h}, \overrightarrow{\mathcal{C}}\rangle ;$ there exists $\mathcal{N}$ such that

$$
\left.\langle\vec{A}, \overrightarrow{\mathcal{B}}, \vec{h}, \overrightarrow{\mathcal{C}}, \mathcal{N}\rangle \in \mathcal{D}, \mathcal{N} \cap M_{1}=\mathcal{N}_{q^{\prime}} \text { and } M_{1} \in \mathcal{N}\right\} .
$$

By the property (u1), $\mathcal{D}^{-} \in H\left(\aleph_{2}\right) \cap M$. We define $\mathcal{E}$ to be the set of $\langle\vec{A}, \overrightarrow{\mathcal{B}}, \vec{h}, \overrightarrow{\mathcal{C}}\rangle \in \mathcal{D}^{-}$for which there exists $\mathcal{N}$ such that:

- $\operatorname{dom}(\vec{A}) \supseteq \operatorname{dom}\left(\vec{A}_{q^{\prime}}\right)$ and for all $\nu \in \operatorname{dom}\left(\vec{A}_{q^{\prime}}\right), \vec{A}(\nu) \supseteq \vec{A}_{q^{\prime}}(\nu), \overrightarrow{\mathcal{B}}(\nu) \supseteq$ $\overrightarrow{\mathcal{B}}_{q^{\prime}}(\nu)$ and $\overrightarrow{\mathcal{C}}(\nu) \supseteq \overrightarrow{\mathcal{C}}_{q^{\prime}}(\nu)$, and $\vec{h} \supseteq \vec{h}_{q^{\prime}}$,

- for all $\nu \in \operatorname{dom}\left(\vec{A}_{q^{\prime}}\right)$ and $B \in \overrightarrow{\mathcal{B}}(\nu)$, there exists $\langle\nu, i\rangle \in \sigma \cup \tau$ such that $B \in \mathcal{F}_{+}^{i}$,

- for all $\langle\nu, i\rangle \in \sigma, \overrightarrow{\mathcal{B}}(\nu)=\overrightarrow{\mathcal{B}}_{q^{\prime}}(\nu)$,

- for all $\langle\nu, i\rangle \in \tau, \overrightarrow{\mathcal{B}}(\nu) \cap M_{1}=\overrightarrow{\mathcal{B}}_{q^{\prime}}(\nu)$ and for all $B \in\left(\overrightarrow{\mathcal{B}}(\nu) \cap \mathcal{F}_{+}^{i}\right) \backslash M_{1}$ and $B^{\prime} \in \overrightarrow{\mathcal{B}}_{q^{\prime}}(\nu) \cap \mathcal{F}_{+}^{i}$ with $B^{\downarrow} \cap M_{1}=\left(B^{\prime}\right)^{\downarrow} \cap M_{1}$, either $B \subseteq B^{\prime}$ or $\Delta\left(B, B^{\prime}\right)>\delta$. 
By the property (u1) again, $\mathcal{E} \in H\left(\aleph_{2}\right) \cap M$, and $\mathcal{E}$ is not empty because the tuple

$$
\left\langle\overrightarrow{A_{q}}, \overrightarrow{\mathcal{B}_{q}}, \overrightarrow{h_{q}}, \overrightarrow{\mathcal{C}_{q}}, \mathcal{N}_{q} \cup\left\{M_{1}\right\}\right\rangle
$$

is in $\mathcal{E}$. So there exists $\left\langle\vec{A}_{r}, \overrightarrow{\mathcal{B}}_{r}, \vec{h}_{r}, \overrightarrow{\mathcal{C}_{r}}\right\rangle \in \mathcal{E} \cap M$, and hence there exists $\mathcal{N}_{r} \in$ $M$ which witnesses that $\left\langle\vec{A}_{r}, \overrightarrow{\mathcal{B}}_{r}, \vec{h}_{r}, \overrightarrow{\mathcal{C}}_{r}\right\rangle \in \mathcal{D}^{-}$. Then $r=\left\langle\vec{A}_{r}, \overrightarrow{\mathcal{B}}_{r}, \vec{h}_{r}, \overrightarrow{\mathcal{C}}_{r}, \mathcal{N}_{r}\right\rangle$ is an element of $\mathcal{D}$ which satisfies (i)-(vi).

Let $r^{\prime}=\left\langle\vec{A}_{r^{\prime}}, \overrightarrow{\mathcal{B}}_{r^{\prime}}, \vec{h}_{r^{\prime}}, \overrightarrow{\mathcal{C}}_{r^{\prime}}, \mathcal{N}_{r^{\prime}}\right\rangle$ be such that:

- $\operatorname{dom}\left(\vec{A}_{r^{\prime}}\right):=\operatorname{dom}\left(\vec{A}_{r}\right), \vec{A}_{r^{\prime}}(\nu):=\vec{A}_{r}(\nu)$ for each $\nu \in \operatorname{dom}\left(\vec{A}_{r^{\prime}}\right) \backslash$ $\operatorname{dom}\left(\vec{A}_{q}\right)$, and $\vec{A}_{r^{\prime}}(\nu):=\vec{A}_{r}(\nu) \cup \vec{A}_{q}(\nu)$ for each $\nu \in \operatorname{dom}\left(\vec{A}_{q}\right)$,

- $\operatorname{dom}\left(\overrightarrow{\mathcal{B}_{r^{\prime}}}\right):=\operatorname{dom}\left(\overrightarrow{\mathcal{B}_{r}}\right), \overrightarrow{\mathcal{B}_{r^{\prime}}}(\nu):=\overrightarrow{\mathcal{B}}_{r}(\nu)$ for each $\nu \in \operatorname{dom}\left(\overrightarrow{\mathcal{B}_{r^{\prime}}}\right) \backslash \operatorname{dom}\left(\overrightarrow{\mathcal{B}_{q}}\right)$, and $\overrightarrow{\mathcal{B}}_{r^{\prime}}(\nu):=\overrightarrow{\mathcal{B}}_{r}(\nu) \cup \overrightarrow{\mathcal{B}}_{q}(\nu)$ for each $\nu \in \operatorname{dom}\left(\overrightarrow{\mathcal{B}}_{q}\right)$,

- $\vec{h}_{r^{\prime}}:=\vec{h}_{r} \cup \vec{h}_{q}$,

- $\operatorname{dom}\left(\overrightarrow{\mathcal{C}_{r^{\prime}}}\right):=\operatorname{dom}\left(\overrightarrow{\mathcal{C}_{r}}\right), \overrightarrow{\mathcal{C}_{r^{\prime}}}(\nu):=\overrightarrow{\mathcal{C}_{r}}(\nu)$ for each $\nu \in \operatorname{dom}\left(\overrightarrow{\mathcal{C}_{r^{\prime}}}\right) \backslash \operatorname{dom}\left(\overrightarrow{\mathcal{C}_{q}}\right)$, and $\overrightarrow{\mathcal{C}_{r^{\prime}}}(\nu):=\overrightarrow{\mathcal{C}}_{r}(\nu) \cup \overrightarrow{\mathcal{C}_{q}}(\nu)$ for each $\nu \in \operatorname{dom}\left(\overrightarrow{\mathcal{C}_{q}}\right)$,

- $\mathcal{N}_{r^{\prime}}=\mathcal{N}_{r} \cup \mathcal{N}_{q}$.

Then as seen above, $r^{\prime}$ is a condition of $\mathrm{Q}\left(\left\langle C_{\nu} ; \nu \in \omega\right\rangle\right) \cap M$, and so is a common extension of $q$ and $r$. Therefore $\mathcal{D} \cap M$ is predense below $p^{+}$.

So Theorem 5 is proved, and we can force $\left({ }^{4}\right)$ that if $\left\langle T^{n} ; n \in \omega\right\rangle$ is a decreasing sequence of $\omega_{1}$-trees with $(\mathbf{u} \mathbf{1 - 2})$ and $\left\langle\pi^{n} ; n \in \omega \backslash\{0\}\right\rangle$ is a sequence of functions with (p1-3), then the space $\mathfrak{X}\left(\left\langle T^{n}, \pi^{n} ; n \in \omega\right\rangle\right)$ does not satisfy (D1), by a countable support iteration as in $[5, \S 4]$. Notice that a similar result on normality is also proved.

5. Concluding remarks. From Lemma 1.4, $\mathrm{MA}_{\aleph_{1}}(\mathrm{rec})$ implies that every Aronszajn tree is special.

Question 5.1. If $T^{0}$ is a special Aronszajn tree, does the space $\mathfrak{X}\left(\left\langle T^{n}, \pi^{n}\right.\right.$; $n \in \omega\rangle)$ satisfy (D1)?

This would refine the result in $\S 2$, but we may not apply the result in $\S 3$, because we have not proved that it is consistent that a Suslin tree forces $\mathrm{MA}_{\aleph_{1}}(\mathrm{rec})$. So the following question is still open.

QUESTION 5.2. Is it consistent that a Suslin tree forces $\mathrm{MA}_{\aleph_{1}}(\mathrm{rec})$ ?

However, by a similar argument to $\S 3$, we can prove that it is consistent that a Suslin tree forces that every Aronszajn tree is special. If the answer

$\left({ }^{4}\right)$ However, to do this from the ground model of ZFC, we have to modify $\mathrm{Q}\left(\left\langle C_{\nu} ; \nu \in \omega\right\rangle\right)$ to have an $\aleph_{2}$-proper isomorphic condition as in [20]. If we suppose that there exists a supercompact cardinal, we do not need to modify our forcing notions. 
to Question 5.2 is affirmative, this refines the result in $\S 3$. In [14], LarsonTodorčević proved that it is consistent that a Suslin tree forces $\mathcal{K}_{2}(\mathrm{rec})$. So a positive answer to the question below would also refine the result in $\S 3$. In [21], Todorčević proved that $\mathcal{K}_{2}$, which is stronger than $\mathcal{K}_{2}(\mathrm{rec})$, implies that every Aronszajn tree is special, so a positive answer to the following question would also refine Todorčević's result.

Question 5.3. Does $\mathcal{K}_{2}(\mathrm{rec})$ imply that every Aronszajn tree is special?

In Section 4, we have considered the space $\mathfrak{X}\left(\left\langle T^{n}, \pi^{n} ; n \in \omega\right\rangle\right)$ for $\left\langle T^{n}\right.$; $n \in \omega\rangle$ having some properties. So the following questions arise:

Question 5.4. Is it consistent (or true under the Proper Forcing Axiom) that for any decreasing sequence $\left\langle T^{n} ; n \in \omega\right\rangle$ of $\omega_{1}$-trees and sequence $\left\langle\pi^{n} ; n \in \omega \backslash\{0\}\right\rangle$ of functions with (p1-3) (or $\left.(\mathbf{p 1} \mathbf{1} \mathbf{4})\right)$, the space $\mathfrak{X}\left(\left\langle T^{n}, \pi^{n}\right.\right.$; $n \in \omega\rangle)$ does not satisfy (D1)?

In Section 3, we have considered the forcing extension with a coherent Suslin tree, which is equivalently strongly homogeneous (see e.g. $[10,12,18]$ ). Suslin trees can be of two types: free and homogeneous. Suppose that a decreasing sequence $\left\langle T^{n} ; n \in \omega\right\rangle$ of Suslin trees is such that for all $n \in \omega$ and $s, t \in T^{n}$, if $s$ and $t$ are incomparable in $T^{n}$, then $\left(T^{n}\lceil s) \times\left(T^{n}\lceil t)\right.\right.$ satisfies the countable chain condition $\left(^{5}\right)$. Then if $\left\langle\dot{\pi}^{n} ; n \in \omega\right\rangle$ is a $T^{0}$-name for a sequence of functions, then $T^{0}$ forces that the space $\mathfrak{X}\left(\left\langle T^{n}, \dot{\pi}^{n} ; n \in \omega\right\rangle\right)$ satisfies (D1). (The proof is essentially the same as Rudin's original proof, or in the extension with $T^{0}$, each $T^{n}$ has a cone which is Suslin, so it is proved.)

QUESTION 5.5. Is it consistent that there exists a decreasing sequence $\left\langle T^{n} ; n \in \omega\right\rangle$ of Suslin trees such that for all $n \in \omega$ and $s, t \in T^{n}$, if $s$ and $t$ are incomparable in $T^{n}$, then $\left(T^{n}\lceil s) \times\left(T^{n}\lceil t)\right.\right.$ satisfies the countable chain condition, and there is a $T^{0}$-name $\left\langle\dot{\pi}^{n} ; n \in \omega\right\rangle$ for a sequence of functions with (p1-3) (or (p1-4)) such that $T^{0}$ forces that $\mathfrak{X}\left(\left\langle T^{n}, \dot{\pi}^{n} ; n \in \omega\right\rangle\right)$ is normal (hence a Dowker space)?

Acknowledgments. I would like to thank Paul B. Larson and Sakaé Fuchino for giving me a motivation for this work. Paul B. Larson told me about the small Dowker space problem and asked whether we need the Suslinness of a tree to introduce Dowker space due to Rudin to be Dowker. For this question, Sakaé Fuchino asked (or suggested) whether Rudin's Dowker space is still Dowker in the extension by the Suslin tree which constructs the space.

$\left(^{5}\right)$ This property is a weak form of freeness. 
I would also like to thank Nobuyuki Kemoto and Haruto Ohta for encouragement, and Stevo Todorčević for letting me know the result due to Bernhard König about a dichotomy of $\omega_{1}$-trees.

I am grateful to the referee for his careful reading and helpful comments and suggestions. In particular, Section 4 comes from a question in the first referee report.

\section{References}

[1] Z. T. Balogh, A small Dowker space in ZFC, Proc. Amer. Math. Soc. 124 (1996), $2555-2560$.

[2] J. E. Baumgartner, Iterated forcing, in: Surveys in Set Theory, London Math. Soc. Lecture Note Ser. 87, Cambridge Univ. Press, Cambridge, 1983, 1-59.

[3] -, Applications of the proper forcing axiom, in: Handbook of Set-Theoretic Topology, North-Holland, Amsterdam, 1984, 913-959.

[4] J. Brendle, Van Douwen's diagram for dense sets of rationals, Ann. Pure Appl. Logic 143 (2006), 54-69.

[5] T. Carlson and R. Laver, Sacks reals and Martin's axiom, Fund. Math. 133 (1989), $161-168$.

[6] H. G. Dales and W. H. Woodin, An Introduction to Independence for Analysts, London Math. Soc. Lecture Note Ser. 115, Cambridge Univ. Press, Cambridge, 1987.

[7] C. H. Dowker, On countably paracompact spaces, Canad. J. Math. 3 (1951), 219-224.

[8] T. Jech, Set Theory. The Third Millennium Edition, Revised and Expanded, Springer Monogr. Math., Springer, Berlin, 2003.

[9] M. Kojman and S. Shelah, A ZFC Dowker space in $\aleph_{\omega+1}$ : an application of PCF theory to topology, Proc. Amer. Math. Soc. 126 (1998), 2459-2465.

[10] B. König, Trees, games and reflections, Ph.D. thesis, Ludwig-Maximilians-Univ. München, 2002.

[11] K. Kunen, Set Theory. An Introduction to Independence Proofs, Stud. Logic Found. Math. 102, North-Holland, Amsterdam, 1983.

[12] P. Larson, An $\mathbb{S}_{\max }$ variation for one Souslin tree, J. Symbolic Logic 64 (1999), 81-98.

[13] P. Larson and S. Todorčević, Chain conditions in maximal models, Fund. Math. 168 (2001), 77-104.

[14] —, 一, Katětov's problem, Trans. Amer. Math. Soc. 354 (2002), 1783-1791.

[15] M. E. Rudin, Countable paracompactness and Souslin's problem, Canad. J. Math. 7 (1955), 543-547.

[16] -, A normal space $X$ for which $X \times I$ is not normal, Fund. Math. 73 (1971), 179-186.

[17] —, Dowker spaces, in: Handbook of Set-Theoretic Topology, North-Holland, Amsterdam, 1984, 761-780.

[18] S. Shelah and J. Zapletal, Canonical models for $\aleph_{1}$-combinatorics, Ann. Pure Appl. Logic 98 (1999), 217-259.

[19] P. J. Szeptycki, Small Dowker spaces, in: Open Problems in Topology II, Elsevier, 2006, 233-239.

[20] S. Todorčević, Directed sets and cofinal types, Trans. Amer. Math. Soc. 290 (1985), $711-723$. 
[21] S. Todorčević, Partitioning pairs of countable ordinals, Acta Math. 159 (1987), 261294.

[22] —, Partition Problems in Topology, Contemp. Math. 84, Amer. Math. Soc., Providence, RI, 1989.

[23] T. Yorioka, Some weak fragments of Martin's Axiom related to the rectangle refining property, Arch. Math. Logic 47 (2008), 79-90.

Department of Mathematics

Shizuoka University

Ohya 836, Shizuoka, 422-8529, Japan

E-mail: styorio@ipc.shizuoka.ac.jp

Received 6 July 200\%;

in revised form 22 May 2008 